\title{
Insectes utiles
}

et nuisibles du fraisier

et du framboisier

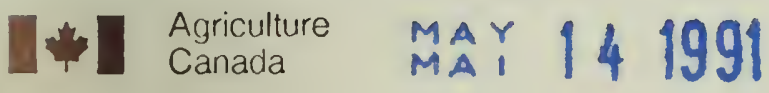

$$
23
$$

Library / Bibliothèque, Ottawa K1A 0C5

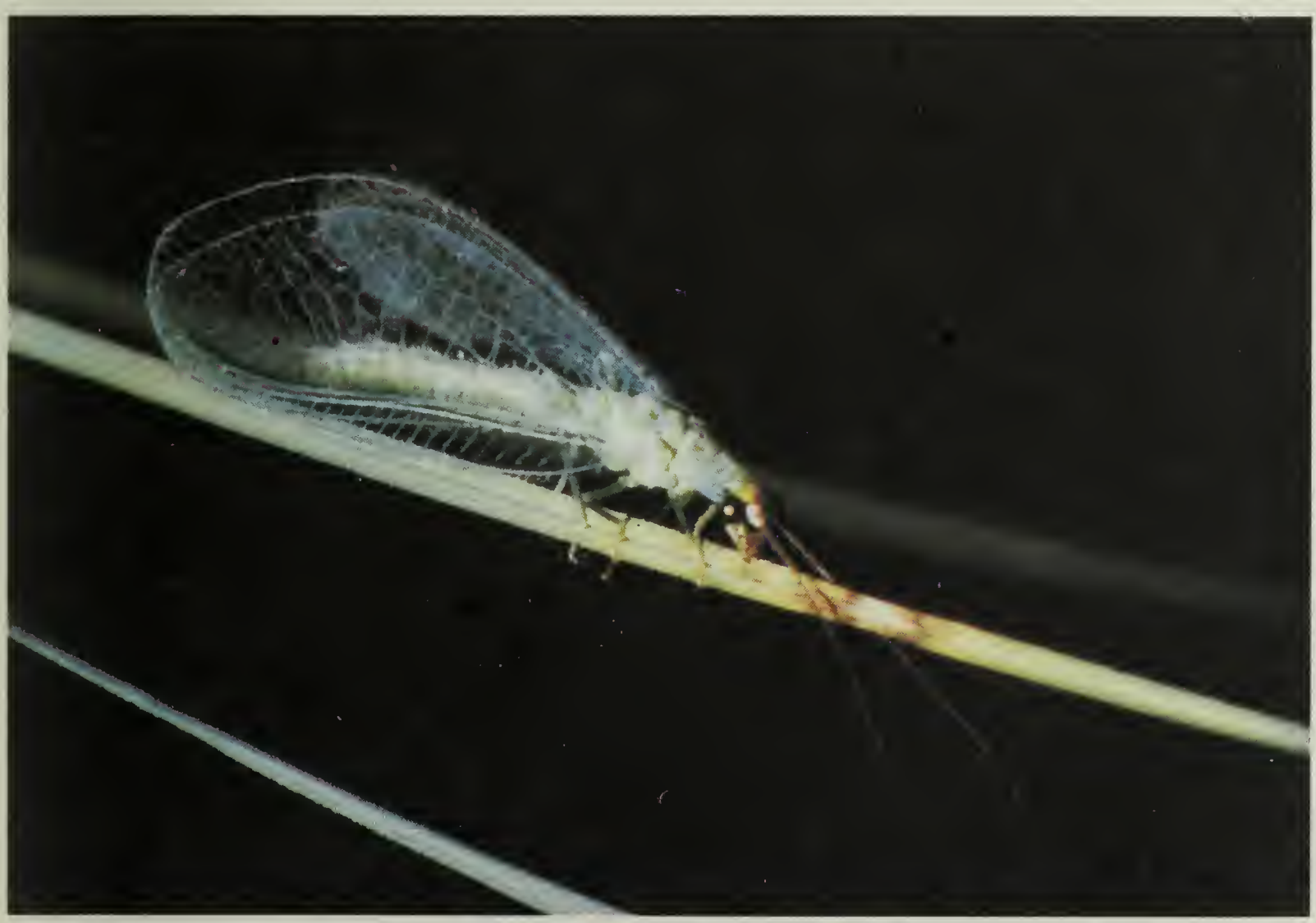

Canadä 



\section{Insectes utiles \\ et nuisibles du fraisier et du framboisier}

Rédigé à contrat par

D.E. Henderson

E.S. Cropconsult Ltd.

Vancouver (Colombie-Britannique)

en collaboration avec

D.A. Raworth

Station de recherches

Vancouver (Colombie-Britannique)

\section{Couverture}

Chrysope à la recherche de pucerons (photo, D. Gillespie)

Agriculture Canada Publication 1863/F

On peut en obtenir des exemplaires à la

Direction générale des communications

Agriculture Canada, Ottawa (Ontario) K1A 0C7

@Ministre des Approvisionnements et Services Canada 1991

$\mathrm{N}^{\circ}$ de cat. A53-1863/1991F ISBN 0-662-96632-5

Imprimé en $19912 \mathrm{M}-04: 91$

Production du Service aux programmes de recherches

Also available in English under the title

Beneficial insects and common pests on strawberry and raspberry crops 
Digitized by the Internet Archive in 2012 with funding from

Agriculture and Agri-Food Canada - Agriculture et Agroalimentaire Canada 


\section{Table des matières}

\section{Remerciements 6}

\section{Introduction 7}

Diptères utiles (mouches) 10

Syrphes (Diptères : syrphidés) 10

Tachinaires (Diptères : tachinidés) 10

Cécidomyies (Diptères : cécidomyiidés) 13

\section{Hyménoptères utiles (guêpes) 13}

Ichneumons (Hyménoptères : ichneumonidés) 13

Braconides (Hyménoptères : braconidés) 14

Chalcidies (Hyménoptères : chalcidoïdés) 15

Coléoptères utiles 15

Coccinelles (Coléoptères : coccinellidés) 15

Staphylins (Coléoptères : staphilinidés) 20

Carabes (Coléoptères : carabidés) 20

\section{Hémiptères utiles 22}

Punaises demoiselles (Hémiptères : nabidés) 22

Réduves (Hémiptères : réduviidés) 22

Punaises tête-de-clou (Hémiptères : lygéidés) 23

Anthocoris (Hémiptères : anthocoridés) 23

Névroptères utiles 25

Chrysopes (Névroptères : chrysopidés) 25

Hémérobies (Névroptères : hémérobidés) 25

Acariens utiles 26

Acariens prédateurs (Acariens : phytoséidés) 26

Autres prédateurs 26

Ravageurs courants du framboisier et du fraisier 27

Punaises et lygides (Hémiptères : miridés) 27

Pucerons (Homoptères : aphididés) 27

Cicadelles et cercopes (Homoptères) 30

Charançons et autres coléoptères (Coléoptères) 31

Enrouleuses, chenilles, noctuelles et perceurs (Lépidoptères) 32

Tenthrèdes (Hyménoptères : tenthrèdinidés) 32

Acariens 33

Bibliographie 34 


\section{Remerciements}

La présente brochure a vu le jour en réponse à une demande des membres de la Lower Mainland Horticultural Improvement Association, formulée en février 1988, lors des mini-cours présentés chaque année aux cultivateurs, à Clearbrooke (C.-B.). Il s'agit en fait d'un projet coopératif. Le Service aux programmes de recherches d'Agriculture Canada s'est occupé de la publication, dont il a absorbé le coût, tandis que la Direction de la protection des cultures du ministère de l'Agriculture et des Pêcheries de Colombie-Britannique a financé la collecte des données et la rédaction du document en sous-traitance par des spécialistes. Enfin, les chercheurs et les professionnels des quatre coins du Canada dont voici la liste ont fourni photographies, spécimens et renseignements sur les insectes de leur région : R. Smith, Vedalia Research, Galiano (C.-B.); W.G. Wellington et S.G. Cannings, Université de la Colombie-Britannique, Vancouver (C.-B.); D. Gillespie, Agriculture Canada, Agassiz (C.-B.); C. Vincent et N. Bostanian, Agriculture Canada, Saint-Jean-sur-Richelieu (Québec); M. Luffman, Agriculture Canada, Bouctouche (N.-B.); R. Tremblay, ministère de l'Agriculture du Nouveau-Brunswick, Fredericton (N.-B.); B.D. Schaber, Agriculture Canada, Lethbridge (Alb.); L. Gilkeson, Applied Bionomics, Sidney (C.-B.); M. Steiner et M. Herbut, Centre environnemental, Vegreville (Alb.); R. Long et M. Mackauer, Université Simon Fraser, Burnaby (C.-B.); C. Chan, W. MacDiarmid, H. Severson et W.T. Cram, Agriculture Canada, Vancouver (C.-B.). Ont prêté leur concours à la correction du manuscrit: $\mathrm{H}$. Gerber et $\mathrm{W}$. Peters, ministère de l'Agriculture et des Pêcheries de la Colombie-Britannique, Abbotsford (C.-B.) et S. Krannitz, Pherotech Ltd., Vancouver (C.-B.). Nos plus sincères remerciements à tous. 


\section{Introduction}

Par crainte de détériorer l'environnement, beaucoup d'agriculteurs freinent leur usage de produits chimiques et cherchent d'autres moyens pour lutter contre les ravageurs et les maladies des cultures. Cette approche, baptisée lutte intégrée, gagne de plus en plus de popularité chaque année au Canada et ailleurs. Avant de l'adopter cependant, il faut connaître les autres méthodes de lutte et savoir comment les mettre en pratique. Ce manuel devrait fournir au moins quelques indications à cet égard. Les insectes utiles qui s'attaquent aux ravageurs sont très sensibles aux pesticides, si bien que peu d'entre eux survivent dans les champs où l'on pulvérise régulièrement des antiparasitaires. C'est aussi pourquoi beaucoup de cultivateurs, et même de vulgarisateurs, les connaissent mal. Un agriculteur qui opte pour la lutte intégrée, de son propre chef ou sur les conseils d'un expert, doit commencer par observer régulièrement la population de ravageurs afin d'évaluer la menace qu'ils posent. Il ne recourra à un insecticide que si les parasites menacent d'augmenter au point de causer des pertes économiques. Alors, la population de nombreux insectes, y compris d'insectes utiles, commencera à s'accroître dans le champ. Il est donc capital de pouvoir identifier les insectes utiles pour les différencier des ravageurs. Même s'il existe plusieurs brochures ou publications sur les ravageurs au Canada, aucune ne porte sur les insectes utiles.

La présente brochure décrit les insectes utiles du fraisier et du framboisier, cependant on retrouvera beaucoup de ces insectes sur d'autres cultures attaquées par les mêmes ravageurs. Ainsi, les prédateurs des pucerons du fraisier détruiront les pucerons, peu importe la plante sur laquelle ils se trouvent. Par ailleurs, si certains insectes utiles (les guêpes parasites par exemple) ne s'attaquent qu'à un seul ravageur, d'autres insectes ayant une forte ressemblance à ceux décrits ici seront les prédateurs d'autres espèces nuisibles. Cette brochure, par conséquent, facilitera non seulement l'identification des insectes utiles du fraisier et du framboisier, mais aussi celle des insectes utiles de la majorité des cultures, en général. 

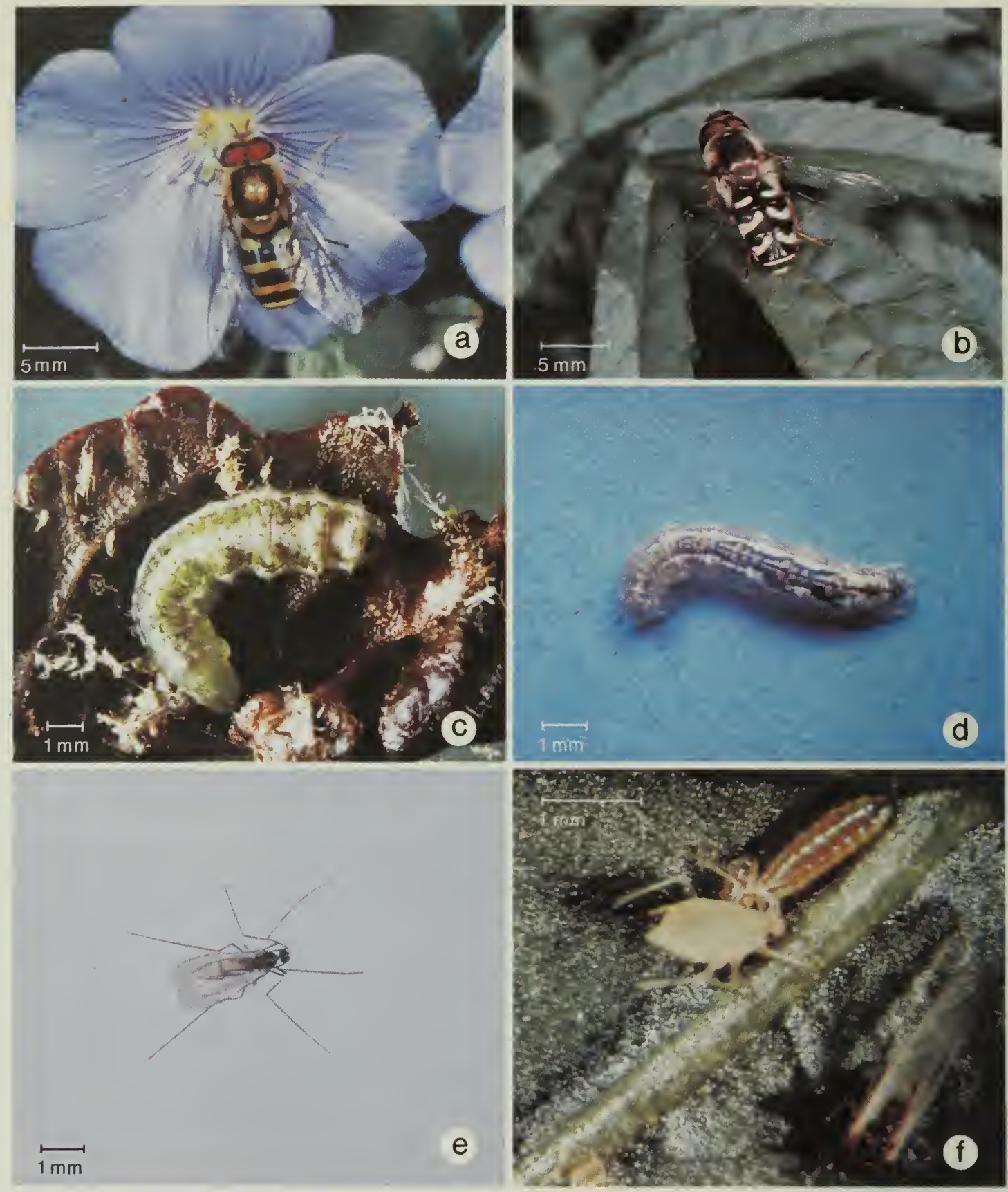

Planche 1 a Syrphe adulte (photo W.G. Wellington)

b Syrphe adulte (photo W.G. Wellington)

c Larve de syrphe (photo C. Chan)

d Larve de syrphe (photo H. Severson)

e Larve de cécidomyie (photo W. McDiarmicl)

$f$ Cécidomyie attaquant un puceron (photo L. Gilkeson) 
Appel aux professionnel Quand nous avons rassemblé l'information et la documentation nécessaires à la rédaction de cet ouvrage, nous nous sommes aperçus que les renseignements manquaient sur la distribution des insectes utiles et que les photos étaient rares. Il est donc possible que les données d'ordre biologique soient incomplètes et que certaines illustrations fassent défaut. Si vous possédez des renseignements sur les insectes utiles de votre région et surtout de bonnes photographies, nous vous serions reconnaissants de communiquer avec nous. Nous avons l'intention de préparer d'autres guides du même genre pour différentes cultures. Toute contribution, sous forme d'information ou de photographies, ne pourra qu'en accroître l'utilité. Il est possible de communiquer avec l'un ou l'autre auteur aux adresses suivantes:

D.E. Henderson, E.S Cropconsult Ltd., 2962 West 15th Ave., Vancouver, B.C. V6K 3A3

D.A. Raworth, Research Station, Research Branch, Agriculture Canada, 6660 N.W. Marine Drive, Vancouver, B.C. V6T 1 X2. 


\section{Diptères utiles (mouches)}

\section{Syrphes (Diptères : syrphidés)}

Les syrphes sont des mouches de 6 à $13 \mathrm{~mm}$ de longueur. Leur abdomen rayé ressemble à celui des guêpes et des abeilles, mais il est dépourvu de dard (planche $1 a$ et $b$ ). Les rayures servent d'avertissement et mettent l'insecte à l'abri d'éventuels prédateurs, par mimétisme. Les nombreuses espèces que compte cette famille sont de bons pollinisateurs, et leur efficacité à ce titre n'est surpassée que par les abeilles. Les syrphes se nourrissent de pollen, de nectar et de miellat (la substance collante et luisante qui macule les plantes près des colonies de pucerons). Leur aptitude à voler sur place, comme de petits hélicoptères, est ce qui les distingue le mieux des abeilles. Les syrphes ou leur larve (le stade immature) se nourrissent exclusivement de pucerons.

La mouche dépose la plupart de ses œufs sur les plantes fortement infestées, parmi les grosses colonies de pucerons. La femelle pond entre 400 et 1000 œufs blancs oblongs qu'elle dépose un à un ou en petits amas, selon l'espèce. Quelques jours plus tard, les œufs éclosent et une minuscule larve semblable à une limace en sort. En se développant, la larve prend la couleur caractéristique de l'espèce. Certaines sont vertes, lignées ou tachées de blanc (planche 1c) tandis que d'autres sont jaunâtres ou brunâtres avec des marbrures (planche $1 d$ ). La larve cherche les pucerons en soulevant le devant du corps et en se balançant latéralement et de haut en bas, pour fouiller la surface des feuilles. Quand elle entre en contact avec un puceron, elle le perce de ses pièces buccales acérées, le soulève et le vide complètement de son contenu. Une larve peut dévorer à elle seule de 200 à 800 pucerons durant sa courte vie de 7 à 10 jours. Parvenue à maturité (elle mesure alors $6 \mathrm{~mm}$ ), la larve se met en quête d'un endroit sombre, une feuille morte enroulée sur la plante par exemple, ou s'enfouit dans le sol. Alors commence la pupaison (stade de repos), qui durera environ 1 semaine. La jeune mouche adulte se mettra à la recherche d'un compagnon dès son émergence et le cycle recommencera. Les syrphes comptent plusieurs générations par année et sont considérés comme un prédateur très efficace des pucerons.

\section{Tachinaires (Diptères : tachinidés)}

Les tachinaires, qui regroupent de nombreuses espèces, ressemblent à la mouche domestique, à l'abeille ou à la guêpe, dont ils ont à peu près la même taille (fig. 1). On les considère comme faisant partie des insectes parasites les plus communs. L'adulte se nourrit des sucs végétaux ainsi que du miellat sécrété par les pucerons, les kermès et les cicadelles. La larve, par contre, parasite d'autres insectes, principalement les formes immatures des papillons nocturnes, des tenthrèdes et des coléoptères, mais aussi quelques punaises et des criquets. Certaines espèces n'attaquent que les chenilles qui vivent cachées, comme les enrouleuses, alors que d'autres 


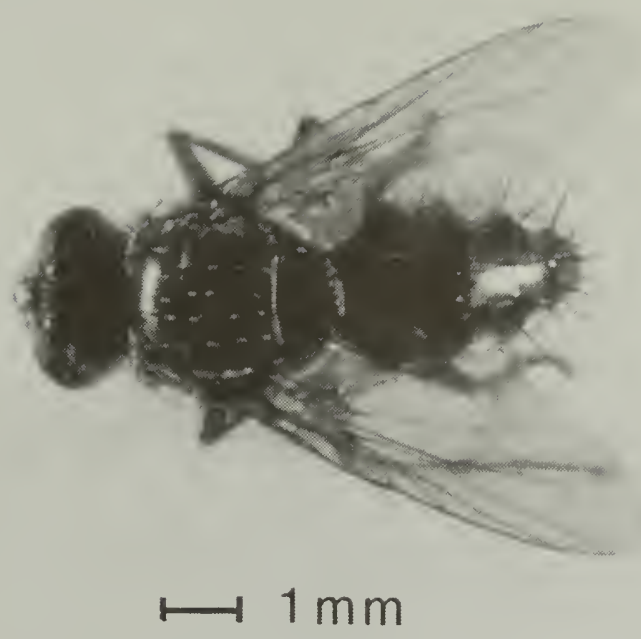

Fig. 1 Tachinaire parasite de l'arpenteuse de framboisier (photo W.T. Cram)

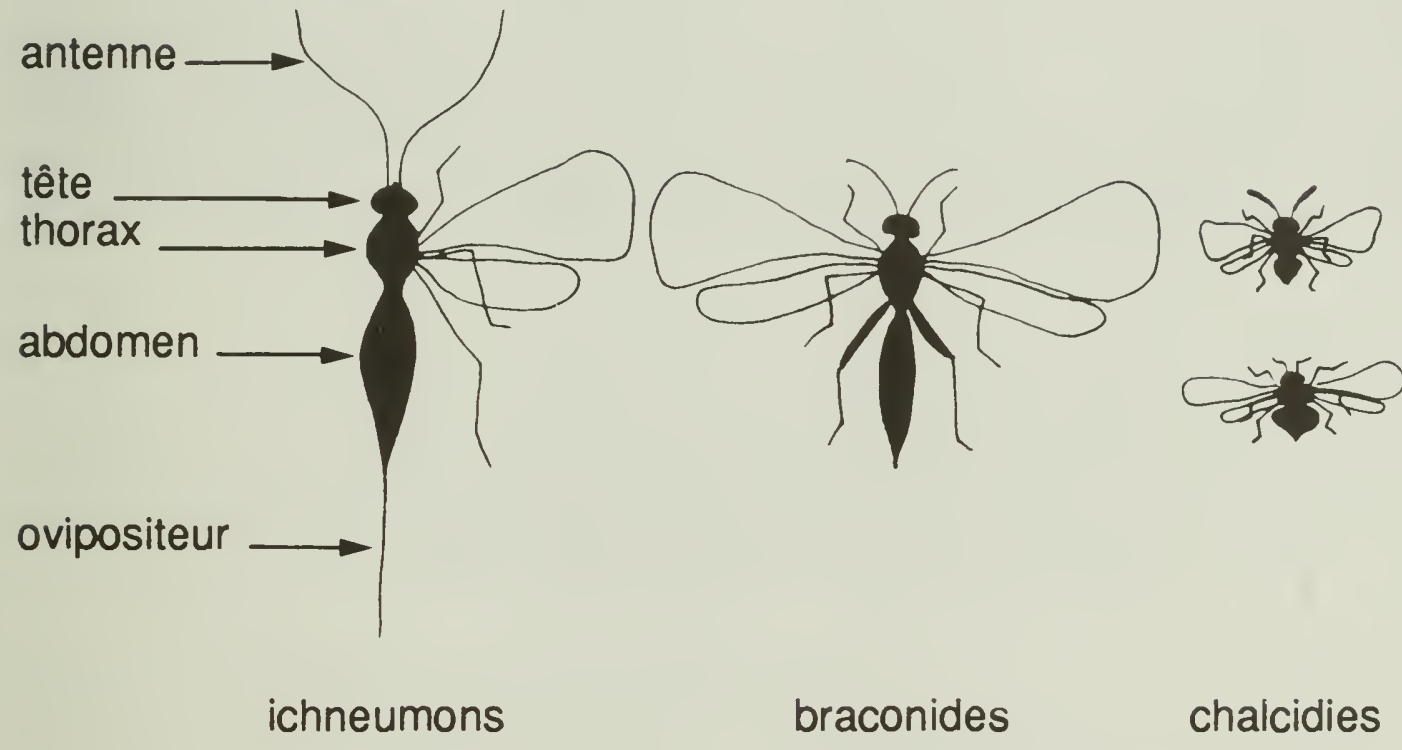

Fig. 2 Formes courantes des ichneumons, des braconides et des chalcidies 

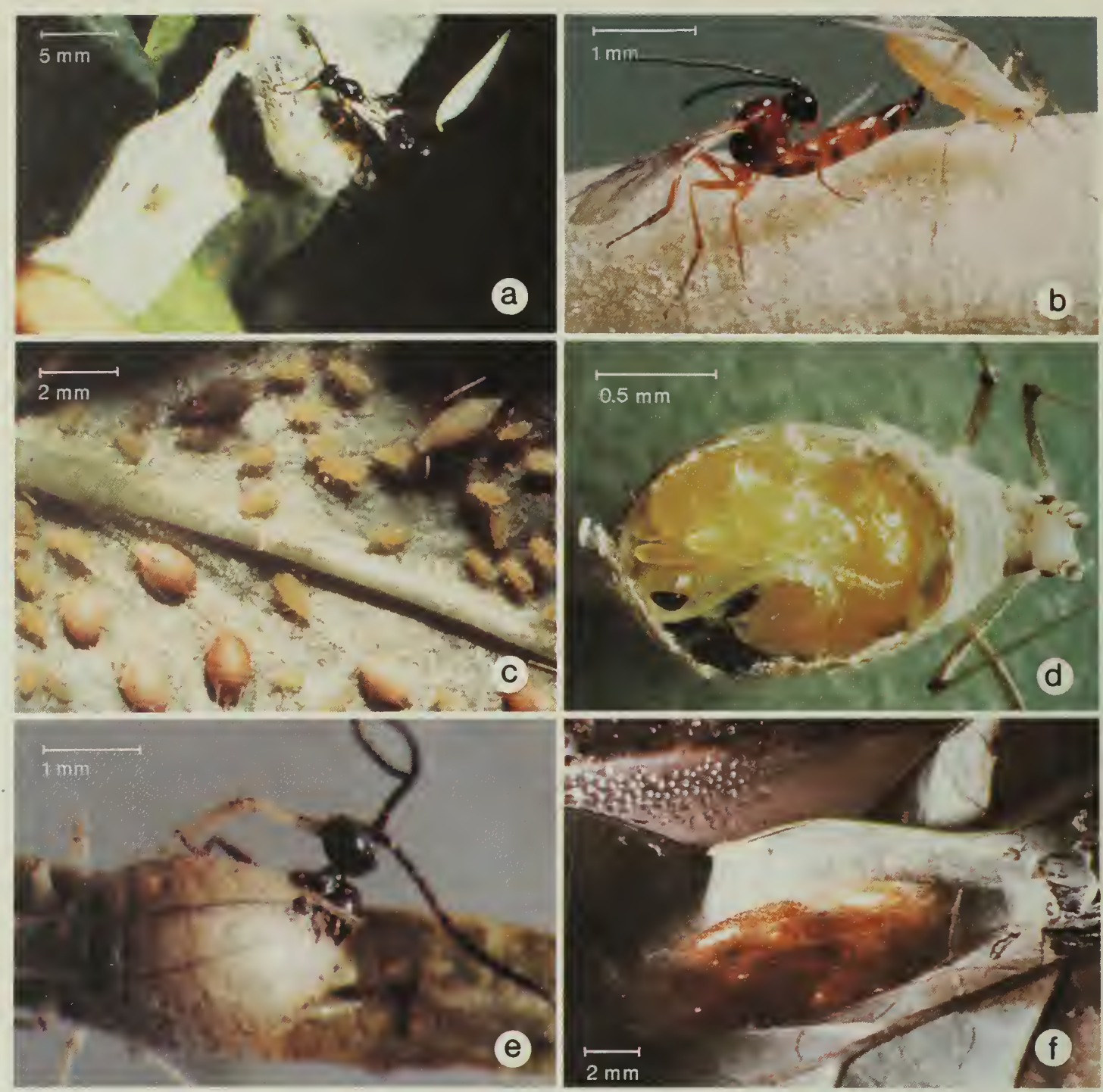

Planche 2 a Ichneumon (Apophua) sur le cocon d'une enrouleuse (photo D. Gillespie)

b Braconide injectant son œuf (photo R. Long)

c «Momies» dans une colonie de pucerons (photo M. Mackauer)

d Pupe d'un braconide dans une momie (photo M. Mackauer)

e Emergence du braconide (photo C. Chan)

f Cocon de braconide (Macrocentrus) (photo D. Gillespie) 
préfèrent les noctuelles. Plusieurs espèces ont été introduites d'Europe pour lutter contre les ravageurs d'Amérique du Nord. C'est notamment le cas du tachinaire de la noctuelle, ennemi commun de ce papillon dans de nombreuses régions du Canada. La femelle dépose ses oufs directement sur l'hôte ou le feuillage qu'il dévorera. Parfois, elle les lui inocule même directement dans le corps. Quelques espèces sont vivipares et laissent les jeunes larves là où d'autres tachinaires déposeraient leurs œufs. Des œufs blancs sur une chenille indiquent que celle-ci est parasitée par un tachinaire. La larve nouvellement éclose se creuse un passage dans l'hôte dont elle dévorera les organes internes plusieurs jours durant, puis abandonnera la dépouille pour entamer la pupaison, à proximité. Tous les insectes parasités par les tachinaires finissent par mourir.

\section{Cécidomyies (Diptères : cécidomyiidés)}

Adulte, la cécidomyie du puceron (Aphidoletes aphidimyza (Rondani)) mesure 2 ou $3 \mathrm{~mm}$ de longueur. Elle est difficile à repérer en raison de ses habitudes nocturnes et de la taille minuscule de son corps délicat (planche 1 e). La femelle vit environ 10 jours et pond près de 250 œufs. Un repas de sève ou de nectar lui permettra de produire un premier lot d'environ 40 œufs. Pour pondre davantage cependant, elle a besoin de miellat de puceron. Contrairement à l'adulte, d'aspect fragile, la petite larve orange ( $1,5 \mathrm{~mm}$ de long) est très robuste (planche $1 f$ ). Elle se nourrit de pucerons et reste à l'extérieur tout l'hiver, dans la plupart des régions du Canada. La larve capture des pucerons en perçant une patte et en inoculant une toxine paralysante; ensuite, elle vide complètement le corps de son contenu. Si les pucerons sont nombreux, la larve en tue plus qu'elle en mange. Après 1 ou 2 semaines de ce manège, elle se laisse choir sur le sol où elle creuse une galerie et commence la pupaison. Dix à 14 jours plus tard émerge un nouvel adulte. Des expériences effectuées en Colombie-Britannique indiquent que la population de cécidomyies du puceron augmente quand les pucerons prolifèrent et diminue lorsqu'ils se font plus rares. On peut acheter au Canada une espèce de cécidomyie qu'on utilise couramment pour combattre les pucerons dans les cultures de serre et les arbres fruitiers. Certains membres de cette famille détruisent aussi les œufs et les larves d'acariens. Enfin, d'autres parasitent les cochenilles et leurs œufs.

\section{Hyménoptères utiles (guêpes)}

\section{Ichneumons (Hyménoptères : ichneumonidés)}

Les ichneumons sont des guêpes au corps élancé qui peuvent mesurer de 5 à $36 \mathrm{~mm}$ de longueur. La femelle porte habituellement au bout de l'abdomen une longue structure similaire à une aiguille (tarière) qu'elle utilise pour déposer ses œufs (fig. 2, p. 11). La tarière est plus longue que le corps et on la prend souvent pour un dard, bien que son rôle consiste à introduire des œufs dans le corps des insectes appelés à nourrir la larve. La larve des ichneumons parasite les stades immatures d'autres insectes, 
surtout des chenilles de papillons nocturnes ou diurnes. La longueur de la tarière permet à la femelle d'inoculer ses œufs dans des hôtes invisibles comme les enrouleuses ou les perce-tiges. Les espèces du genre Apophua en sont un parfait exemple puisqu'elles s'attaquent à la tordeuse du fraisier (Ancylis comptana (Froelich)) alors que celle-ci se cache à l'intérieur de la feuille (planche $2 a$, p. 12). Beaucoup d'ichneumons sont relativement courants au Canada. L'adulte peut être brun, rouge ou noir et porter différentes marques. Il se distingue des autres guêpes parasites par sa grande taille et la longueur de son abdomen, qui dépasse celle de la tête et du thorax réunis. Il possède également une longue tarière.

\section{Braconides (Hyménoptères : braconidés)}

Les braconides sont plus petits que les ichneumons (soitjusqu'à 12 mm de longueur). Leur corps plus court est également plus trapu (fig. 2). Les espèces de cette famille possèdent également une tarière, mais celle-ci reste cachée dans le corps dont elle ne sort qu'au moment de l'inoculation. Les braconides parasitent une plus vaste gamme d'insectes que les ichneumons, les hôtes allant de diverses chenilles comme la tordeuse des citrus (Argyrotaeni citrana (Fernald)) à des mouches, des guêpes, des coléoptères et des pucerons. Lorsque la femelle a injecté son œuf, il sort de celui-ci une larve qui dévorera lentement l'hôte. La larve parvient à maturité quand meurt ce dernier. Elle entame sa pupaison à l'intérieur de la dépouille ou près de celle-ci, quelque fois dans un cocon soyeux d'où émergera la guêpe adulte. A l'instar des ichneumons, on trouve de nombreuses espèces de braconides un peu partout au Canada.

Les braconides parasites des pucerons constituent un groupe important d'insectes utiles du fraisier et du framboisier. En effet, les parasites des pucerons comptent beaucoup de braconides ayant un cycle biologique analogue. La femelle inocule son œuf dans un jeune puceron avec la rapidité de l'éclair (planche $2 b$ ) pendant que celui-ci pompe la sève de la plante. En règle générale, l'attaque dérange le puceron dans son travail sans toutefois l'inciter à quitter la plante sur laquelle il se trouve. Le puceron parasité ne se reproduit habituellement pas, mais continue de se nourrir jusqu'à ce qu'il ait été entièrement dévoré de l'intérieur. La larve adulte fixe la carcasse du puceron à la surface de la feuille et la dépouille prend un aspect parcheminé ou noirâtre. On appelle les pucerons morts de cette façon des "momies". Ils sont très faciles à reconnaître dans une colonie de pucerons vivants (planche $2 c$ ). La momie protège le braconide durant la pupaison (planche $2 d$ ). À la fin de cette dernière, environ une semaine plus tard, la guêpe adulte découpe un trou circulaire dans la momie et sort au grand jour (planche $2 e$ ). La momie vide, percée d'un trou, reste sur la feuille. La présence de momies dans une colonie indique que beaucoup de pucerons sont parasités, même s'ils paraissent en bonne santé, et mourront au bout de quelques jours.

Un autre grand groupe de braconides parasitent les chenilles. Leur cycle biologique reproduit largement celui des braconides qui s'attaquent aux pucerons. Les espèces du genre Copidosoma font toutefois bande à 
part. En effet, la femelle adulte pond son œuf dans un œuf de l'hôte. À mesure que celui-ci traverse les différents stades de son cycle, l'œuf du braconide se divise pour donner plusieurs petites larves. À la mort de l'hôte, les larves entament la pupaison et donneront naissance à plusieurs guêpes adultes. Par cet artifice, la femelle parvient à produire une progéniture nombreuse, même si elle ne trouve qu'un hôte. D'autres braconides, les espèces du genre Macrocentrus par exemple, tirent parti d'un hôte de grande taille comme une chenille en lui injectant un grand nombre d'œufs. Les larves grandissent en dévorant la chair de la chenille. Lorsque celle-ci meurt, on trouve regroupées dans le reste de son cocon les pupes du parasite (planche 2f).

\section{Chalcidies (Hyménoptères : chalcidoïdés)}

La plupart des guêpes de ce groupe sont encore plus petites que les ichneumons et les braconides. D'un bleu ou d'un vert métallique foncé, elles ne mesurent au maximum que $7 \mathrm{~mm}$ de longueur et ont des ailes translucides. La guêpe paraît plutôt sauter que prendre son envol. On trouve les chalcidies partout, mais on a tendance à ne pas les remarquer en raison de leur taille minuscule. Les membres de cette famille parasitent les chenilles des papillons nocturnes et diurnes, les larves de certaines mouches et celles de quelques coléoptères. Les chalcidies ne sont pas toutes utiles, car quelques-unes s'attaquent aux tachinaires et aux pupes des ichneumons.

Un groupe bien connu, le genre Trichogramma, pond ses œufs dans ceux de nombreuses espèces de papillons nocturnes et diurnes. L'adulte, qui mesure moins de $1 \mathrm{~mm}$ de longueur, montre une grande efficacité comme agent de lutte biologique. Il est possible de s'en procurer dans le commerce et on s'en sert sur diverses cultures pour détruire les chenilles, quoique moins fréquemment au Canada qu'aux États-Unis.

\section{Coléoptères utiles}

\section{Coccinelles (Coléoptères : coccinellidés)}

\section{Coccinelles}

La coccinelle adulte est sans doute le prédateur le plus facile à identifier dans un champ. Ses taches rouge vif et noires ressortent de façon frappante sur le vert du feuillage (planche $3 a$, p.16). En outre, comme elle est active le jour, la coccinelle se repère aisément. L'espèce la plus familière, de forme ovale, mesure de 6 à $8 \mathrm{~mm}$ de longueur, a le ventre plat et le dos arrondi. La femelle dépose un amas de 10 à 50 œufs jaune orange en forme de fuseau (planche $3 b$ ) sur les feuilles, près des pucerons. La larve ne ressemble pas du tout à l'adulte. Ses courtes pattes dépassent du corps allongé, souvent foncé, mais agrémenté de marques vives (planches $3 c$ et $d$ ). La larve passe sa vie dans les colonies de pucerons. Quoi qu'elles préfèrent les pucerons, la coccinelle adulte qu'on trouve sur le fraisier et le framboisier et sa larve 

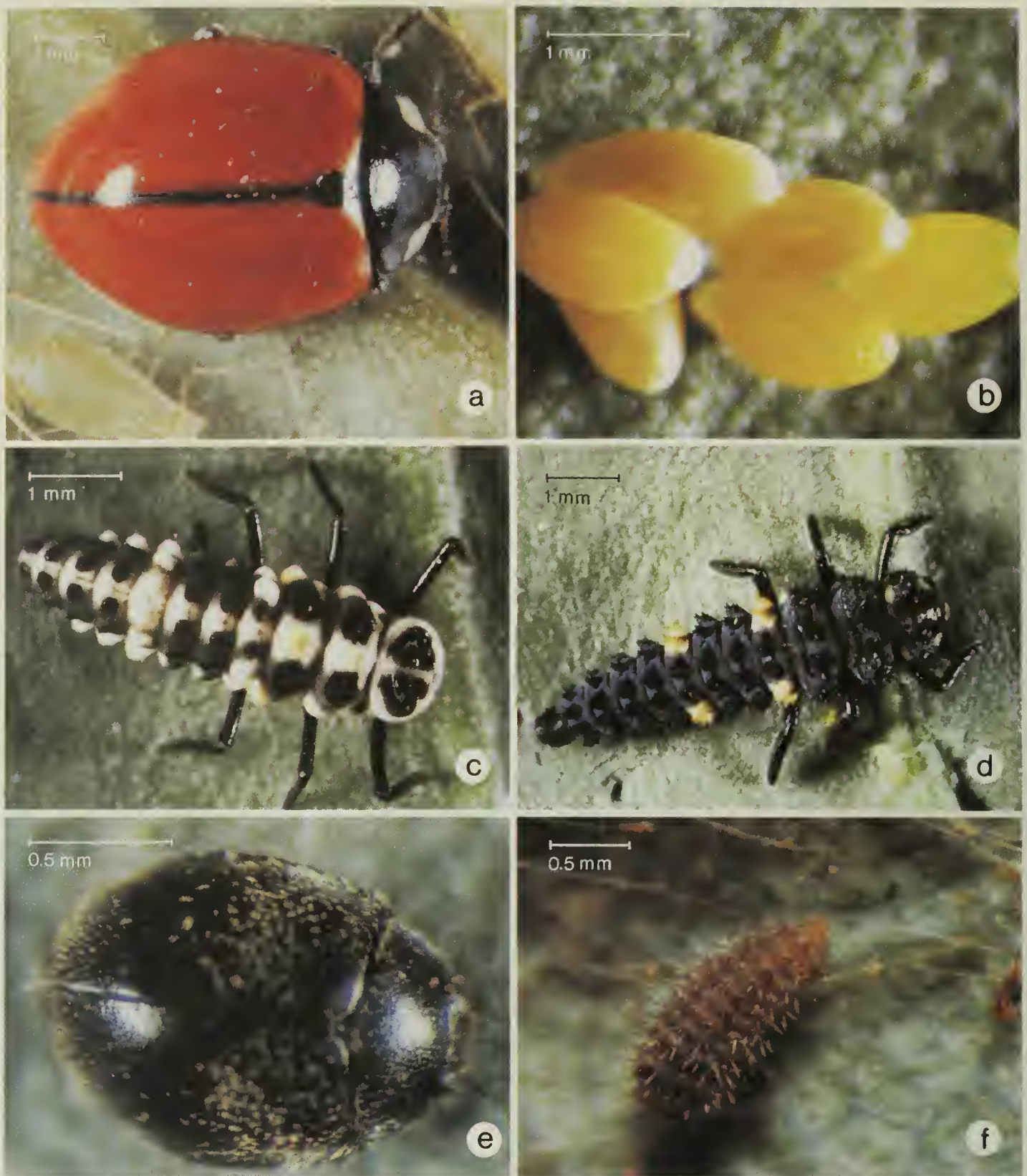

Planche 3 a Coccinelle adulte (photo C. Chan)

b Oeuf de coccinelle (photo C. Chan)

c Larve de coccinelle (photo C. Chan)

d Larve de coccinelle (photo C. Chan)

e Stethorus adulte (photo C. Chan)

$f$ Larve de stethorus (photo C. Chan) 
chercheront d'autre nourriture si les pucerons se font rares, par exemple de petites chenilles, de jeunes punaises, du miellat ou du nectar. Faute d'autres sources d'aliments, la coccinelle est souvent atteinte de cannibalisme. Le nombre de pucerons dévorés chaque jour dépend approximativement de la taille du coléoptère. À maturité, la larve en détruit près de 50 par jour. La femelle adulte doit en manger environ une centaine avant de commencer à pondre et elle en dévorera au moins 2400 durant sa vie qui dure 1 ou 2 mois. L'adulte broie sa victime et ne laisse que les parties les plus coriaces de la carapace. Habituellement, la larve perfore cette dernière et aspire les liquides du corps. L'espèce la plus commune à se nourrir de pucerons est aussi la plus prolifique, car elle pond plusieurs centaines d'œufs à la fois. La coccinelle adulte se déplace beaucoup et on en a vu converger vers les endroits où les pucerons ont atteint une grande densité. Dans ce cas, elle s'avère particulièrement efficace, surtout par temps chaud. Toutefois, quand le nombre de pucerons diminue, les larves, incapables de voler, périssent faute de pouvoir gagner un autre endroit. Elles meurent littéralement de faim.

Le cycle biologique dure de 20 à 35 jours et, en climat tempéré, il n'est pas rare de compter deux ou trois générations par année. L'adulte traverse l'hiver dans des endroits abrités, souvent en compagnie de beaucoup de ses congénères. À l'automne, il est fréquent de voir un grand nombre de coccinelles adultes se rassembler sur le sol ou sur un bâtiment en quête d'un endroit pour hiverner. Toutes les régions du Canada comptent plusieurs espèces de coccinelles qui sont des prédateurs naturels très efficaces du puceron.

\section{Stethorus}

D'autres coccinellidés, les espèces du genre Stethorus par exemple, sont des prédateurs particulièrement voraces qui s'attaquent à tous les stades du tétranyque. L'adulte mesure de 1 à $1,5 \mathrm{~mm}$ de longueur et son corps hémisphérique est noir luisant (planche $3 e$ ). Il est beaucoup moins facile à repérer que les coccinelles rouges, très évidentes, mais on l'aperçoit facilement à l'œil nu quand il se met en quête de tétranyques sur les feuilles de fraisier et de framboisier. La larve brune, allongée, mesure environ $2 \mathrm{~mm}$ de longueur à maturité et porte des touffes de poil ici et là (planche $3 f$ ). La pupe, de forme globulaire, a une carapace robuste et noire d'environ $1 \mathrm{~mm}$ de longueur. Elle se fixe à la surface de la feuille par une extrémité. Le coléoptère adulte est doté de pièces buccales qui lui permettent de dévorer sa proie en entier, alors que la larve se contente d'en sucer le contenu pour ne laisser derrière elle qu'une coquille vide. L'adulte peut détruire de 20 à 60 tétranyques par jour et pondre quotidiennement jusqu'à 10 œufs pendant 1,5 à 3 mois. Il faut de 2 à 4 semaines selon la température pour que l'œuf donne un adulte. Au début de sa vie, la larve consomme moins de tétranyques, mais vers la fin, elle peut en dévorer jusqu'à 250 par jour. Diverses espèces de ce groupe existent un peu partout au Canada. 

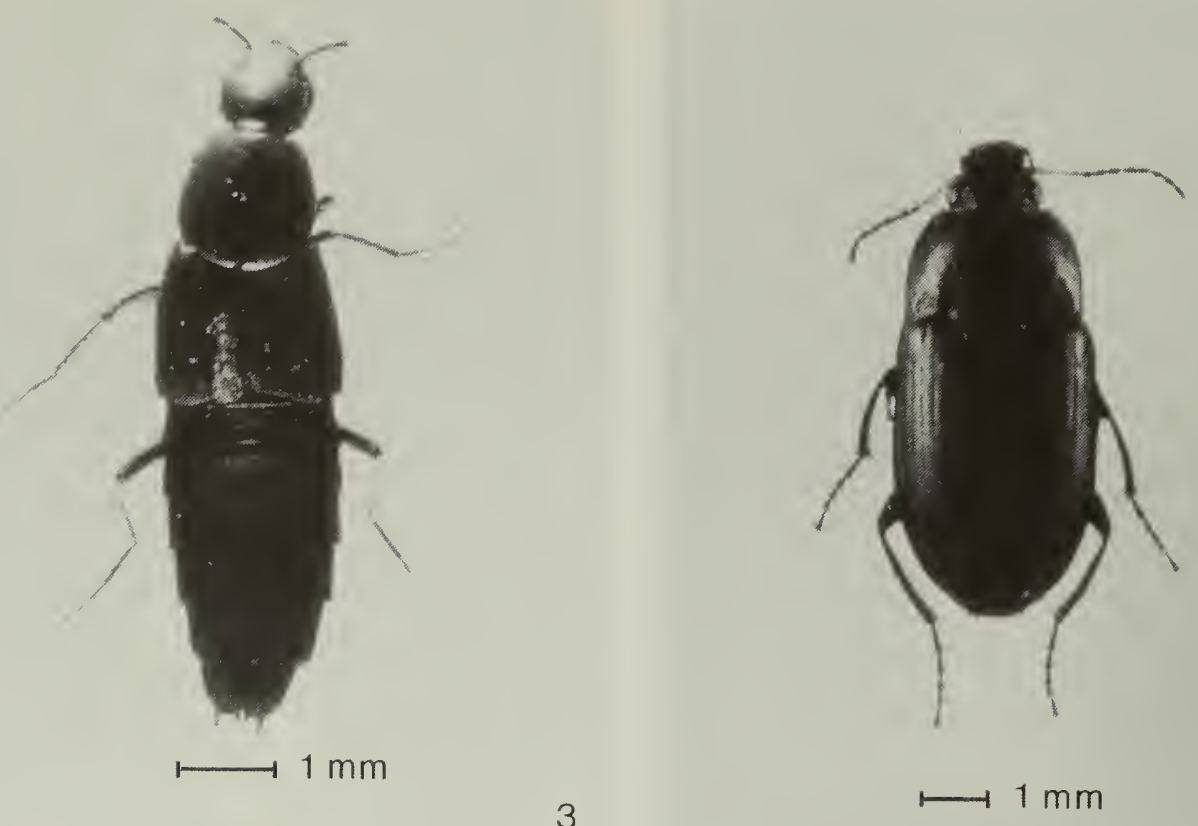

4
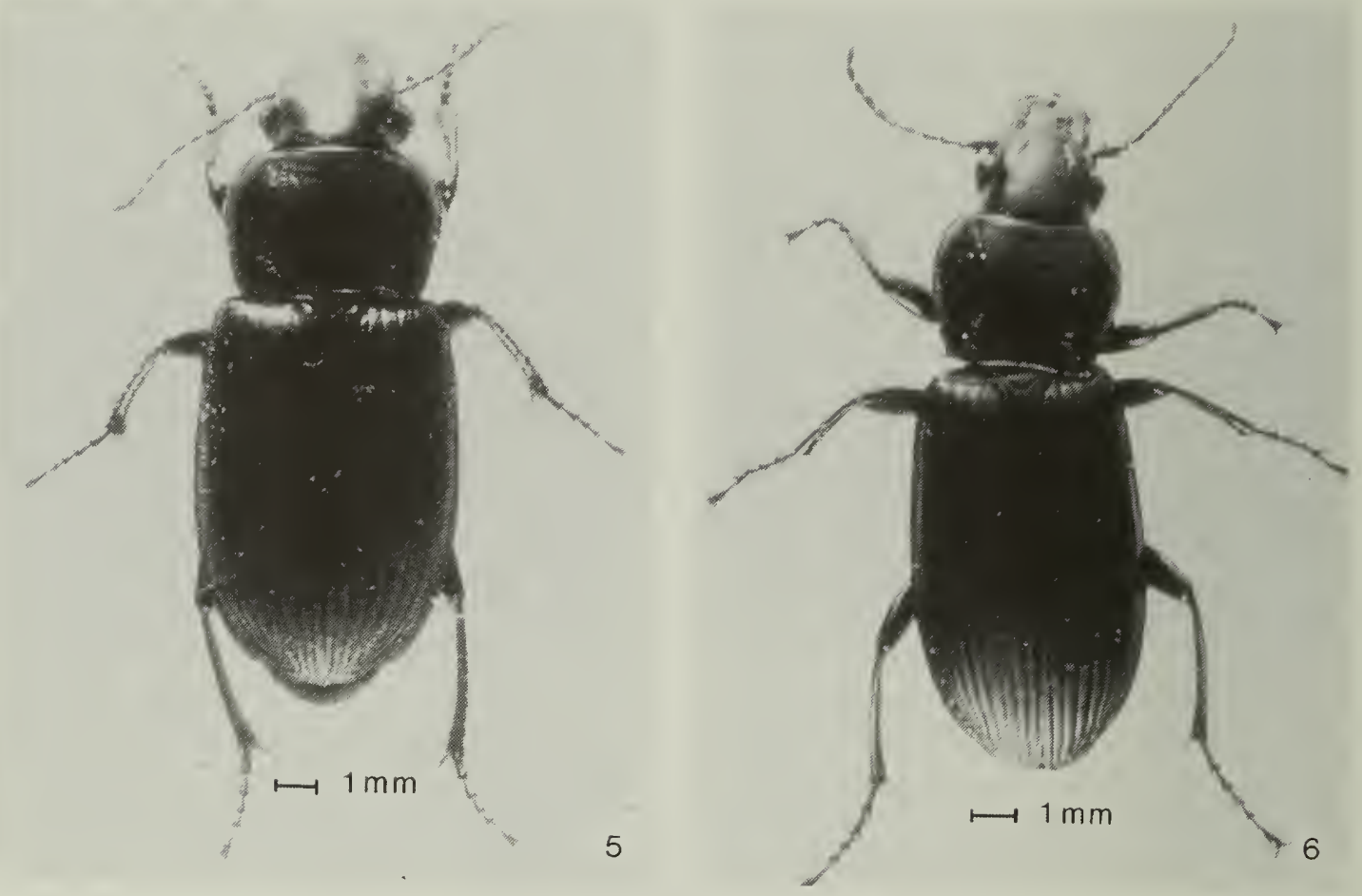

Fig. 3 Staphylin adulte (photo C. Chan)

Fig. 4-6 Carabe adulte (photo H. Severson) 


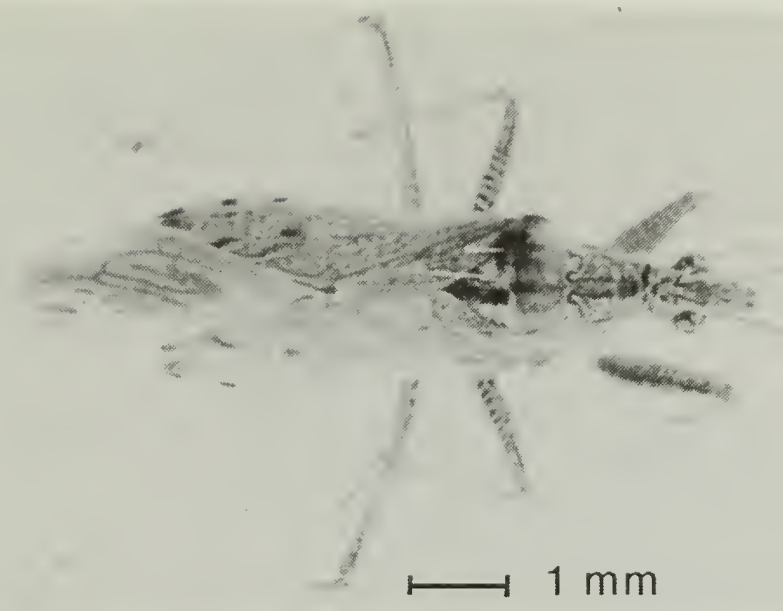

Fig. 7 Odonate adulte (photo B.D. Schaber)

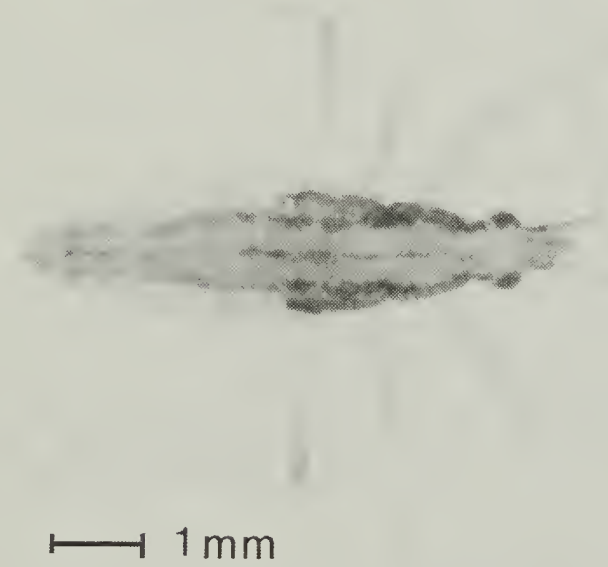

Fig. 8 Nymphe d'odonate (photo B.D. Schaber)

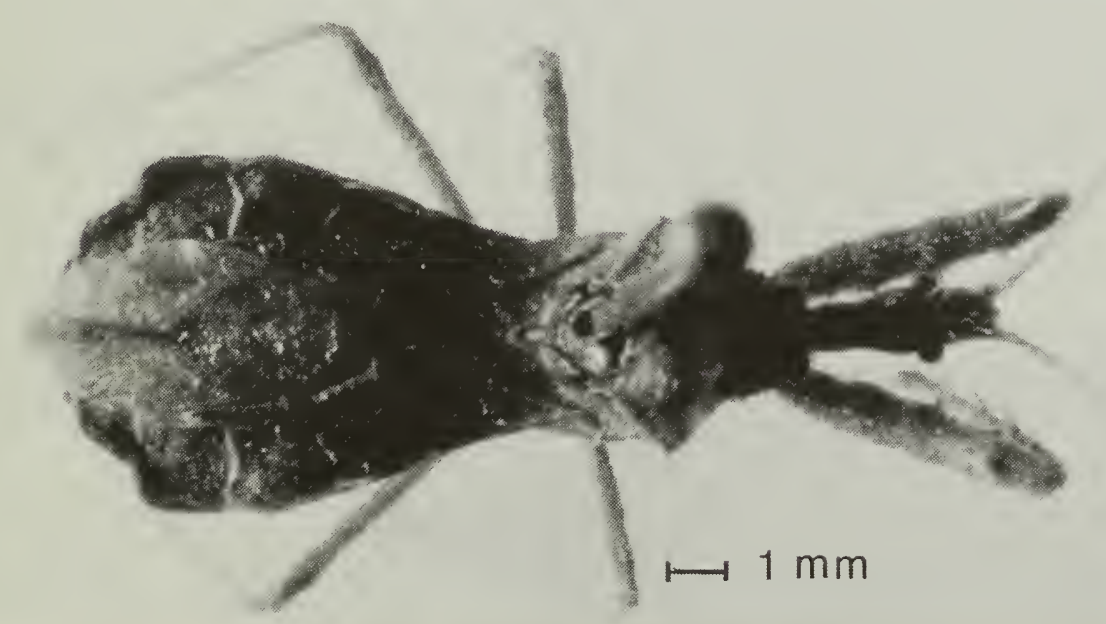

Fig. 9 Réduve adulte (photo C. Chan) 


\section{Staphylins (Coléoptères : staphilinidés)}

Le staphylin adulte peut être noir ou brun et sa taille varie entre 1,5 et $25 \mathrm{~mm}$. Le corps a une forme caractéristique, propre aux staphylins, peu importe leur taille : longue et élancée avec de courts élytres (fig. 3, p. 18). Les ailes sont repliées sous les petits élytres et l'abdomen se prolonge considérablement derrière elles. Le staphylin a pour habitude de replier l'abdomen vers le haut quand on le dérange, comme le font les scorpions. L'insecte porte typiquement la tête légèrement repliée vers le bas, sous le corps. Il s'agit de coléoptères très actifs qui s'enfuient ou s'envolent rapidement. La femelle enfouit ses œufs dans le sol ou les débris végétaux en décomposition. La larve ressemble un peu à l'adulte. Maintes espèces de ce groupe s'attaquent à d'autres insectes, surtout les œufs et les larves dans le sol, mais elles détruisent également les oufs et les larves des phytoparasites. Un petit nombre d'espèces s'attaquent aux tétranyques. L'adulte comme la larve sont très actifs et s'avèrent des prédateurs très agressifs. Les staphylins constituent une vaste famille qu'on retrouve sur tout le continent et regroupent au-delà de 800 espèces, rien qu'au Canada.

Un groupe particulier, les espèces du genre Tachyporus, qu'on trouve aux quatre coins du Canada, préfère attaquer les pucerons qui habitent les plantes basses comme le fraisier. Durant le jour, le coléoptère reste sur le sol, mais la nuit venue, il grimpe dans les plantes à la recherche des pucerons.

\section{Carabes (Coléoptères : carabidés)}

Les carabes (fig. 4 à 6, p. 18) épousent de multiples formes, tailles et couleurs. La majorité des espèces sont bleues, noires ou brun métallique, ont le corps légèrement aplati et de longs élytres finement nervurés de façon longitudinale. La taille varie de quelques millimètres à quelques centimètres. Comme les staphylins, la majorité des carabes se cachent dans le sol, sous les débris végétaux ou dans des endroits similaires durant le jour et ne commencent à s'activer que la nuit. Lorsqu'ils sont dérangés, ils fuient rapidement, mais s'envolent rarement. Presque toutes les espèces sont des prédateurs utiles. La larve, allongée et plus pâle, est munie parfois de grosses mâchoires en forme de pinces au bout d'une grosse tête. On la trouve dans le même habitat que l'adulte et, comme lui, elle s'attaque à d'autres insectes. Le cycle biologique dure souvent un an et quelques espèces parmi les plus grandes vivent plusieurs années. Les plus gros carabes, souvent ceux qui ont les couleurs les plus vives, attaquent les chenilles et, à l'occasion, les charançons adultes, tandis que les espèces plus petites dévorent les œufs, les petites chenilles et d'autres ravageurs. Une grosse espèce commune au Canada (Carabus nemoralis Müller) préfère les limaces et les escargots. On a recensé plus de 800 espèces de carabes au Canada. 

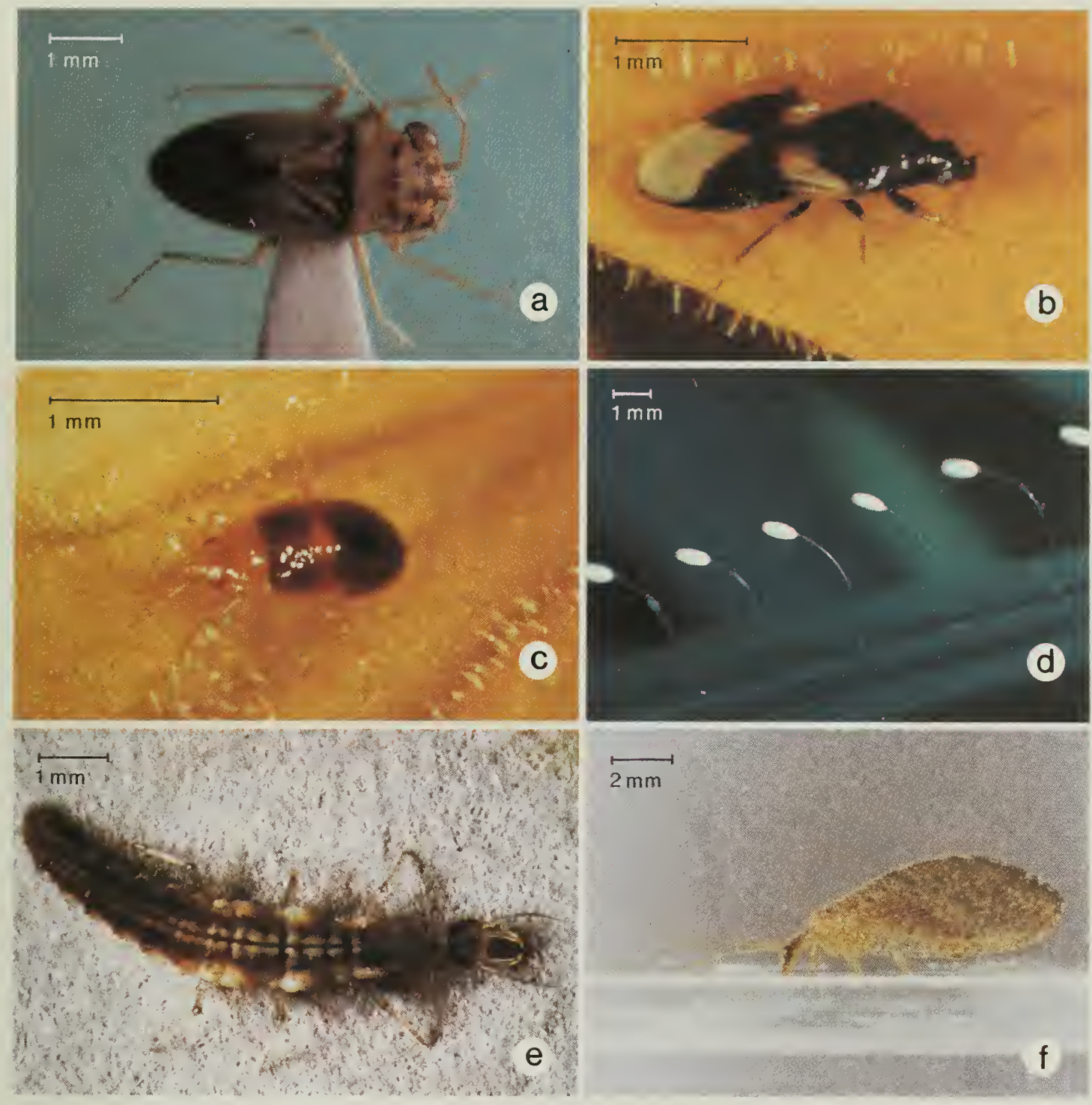

Planche $4 a$ Punaise des bois adulte (photo C. Chan)

b Anthocoris adulte (photo $M$. Herbut)

c Nymphe d'anthocoris (photo M. Herbut)

d Oeufs de chrysope (photo R. Smith)

e Larve de chrysope (photo C. Chan)

$f$ Hémérobie adulte (photo M. MacDiarmid) 


\section{Hémiptères utiles}

\section{Punaises demoiselles (Hémiptères : nabidés)}

La punaise demoiselle est un insecte au corps long, élancé et brun pâle qui peut mesurer jusqu'à $10 \mathrm{~mm}$ de longueur (fig. 7, p. 19). Il est doté de longues pattes dont la première paire est adaptée pour la capture des proies. Ce prédateur affectionne les plantes basses comme les graminées et le fraisier, où il se nourrit des insectes à corps mou qui vivent sur le feuillage, notamment les pucerons, les cicadelles et certaines chenilles, de même que les œufs de papillons nocturnes. Rapide et agressif, la punaise demoiselle attrape même les nymphes de lygide beaucoup plus grosses. La nymphe ressemble à l'adulte par sa forme, mais est beaucoup plus petite et de couleur plus pâle (fig. 8 , p. 19). Elle a également un comportement de prédateur.

En laboratoire, on a vu un odonate adulte dévorer quatre nymphes de lygide par jour. Une fois piquée par les pièces buccales acérées, la victime reste paralysée à jamais, même si elle ne se fait pas dévorer. Au repos, les longues pièces buccales broyeuses restent plaquées sur la face ventrale du corps. La punaise demoiselle ressemble au réduve et on le retrouve couramment au Canada. Ce genre a récemment attiré l'attention des chercheurs en raison de la possibilité de son utilisation comme agent de lutte biologique.

\section{Réduves (Hémiptères : réduviidés)}

Il s'agit d'une famille d'insectes presque tous prédateurs, qui s'attaquent à une vaste gamme de phytoparasites, notamment les pucerons, les cicadelles et les chenilles. Il arrive cependant qu'ils inscrivent à leur tableau de chasse des insectes utiles comme la larve des coccinelles. Le réduve mesure habituellement plus de $10 \mathrm{~mm}$ de long, avec un corps étroit et des pattes filiformes (fig. 9, p. 19). Il est parfois difficile de le différencier des odonates, sauf par la taille, plus importante, et par la couleur, plus foncée. En règle générale, le réduve adulte est noir ou brun, mais la nymphe arbore parfois des couleurs vives. Tous les stades sont prédateurs. La femelle pond habituellement ses œufs dans le sol. En règle générale, on ne compte qu'une génération par année, mais certaines espèces prennent plusieurs années pour compléter leur cycle. Ainsi, quelques espèces peuvent hiverner simultanément sous forme d'œuf, de nymphe ou d'adulte. On trouve des réduves dans toutes les régions du Canada.

Le réduve attaque sa proie en la poignardant avec sa longue pièce buccale légèrement recourbée. Au repos, le réduve ne garde pas son appareil buccal aussi près du corps que l'odonate. Certaines espèces paralysent leur proie avant de la dévorer. 


\section{Punaises tête-de-clou (Hémiptères : lygéidés)}

La punaise tête-de-clou adulte (espèces du genre Geocoris) mesure environ $4 \mathrm{~mm}$ de longueur et $2 \mathrm{~mm}$ de largeur. De couleur gris rosé à noir, elle se remarque par ses deux gros yeux qui dépassent de chaque côté de la tête (planche $4 a, p .21$ ). L'espèce traverse différentes phases de couleur, ce qui signifie que le motif de la carapace peut varier entre les membres de la même espèce. Les œufs, blanc rosé, sont déposés en amas sur la face inférieure des feuilles. La nymphe ressemble à l'adulte en plus petit. L'adulte et la nymphe se laissent tomber sur le sol quand on les dérange. Ils s'attaquent à tous les stades du tétranyque, du puceron et de la cicadelle. Ils se nourrissent des œufs et des jeunes nymphes de lygide et de punaise terne (Lygus lineolaris (Palisot de Beauvois)), à raison de deux à quatre par jour. L'espèce la plus courante, (Geocoris bullatus Say), existe un peu partout au Canada.

On trouve les punaises des bois dans les champs, au printemps, lorsque la population de lygides atteint un point où elle pourrait entraîner de grandes pertes économiques. On les confond souvent avec les lygides, surtout au stade de nymphe. Pourtant si la forme du corps est similaire, la punaise des bois, avec ses grands yeux et sa couleur brun clair, se distingue aisément des nymphes de lygide, de couleur verte et aux yeux petits. En outre, l'adulte et la nymphe de punaise des bois se laissent choir sur le sol quand on les dérange tandis que la lygide s'enfuit. La nymphe et la punaise des bois adulte sont pourvues de pièces buccales qui leur permettent de percer et de sucer les nymphes de lygide et d'autres proies sans carapace. Depuis quelques années, ces prédateurs ont acquis une certaine résistance aux pesticides utilisés pour détruire les lygides (surtout dans les régions où ce ravageur s'attaque à la luzerne). Ils deviennent donc de plus en plus communs et utiles.

\section{Anthocoris (Hémiptères : anthocoridés)}

L'anthocoris est une petite punaise ne mesurant que $2 \mathrm{~mm}$, au corps légèrement aplati et ovale. Ses ailes sont noir et blanc (planche $4 b$ ). Les œufs passent inaperçus, car ils sont partiellement enfouis dans la tige, la nervure principale ou le pétiole des feuilles. Les pièces buccales imitent un long bec pointu avec lequel l'insecte perce et empale sa proie. La nymphe, qui ressemble à l'adulte par sa forme, est de couleur rose jaunâtre pâle à brun et s'avère très active (planche $4 c$ ). La nymphe et l'adulte dévorent tous les stades du tétranyque de même que les thrips, les pucerons et les petites chenilles. La nymphe mange environ 33 tétranyques par jour. Comme l'adulte passe l'hiver à l'abri dans des endroits couverts, il réapparaît très tôt au printemps. Trois ou quatre générations peuvent ainsi se succéder chaque saison. Au Canada, l'anthocoris est le plus commun des prédateurs sur de nombreuses cultures, notamment le fraisier et le framboisier. On peut d'ailleurs s'en procurer dans le commerce et à l'heure actuelle, on s'en sert pour lutter contre les thrips dans les serres. 

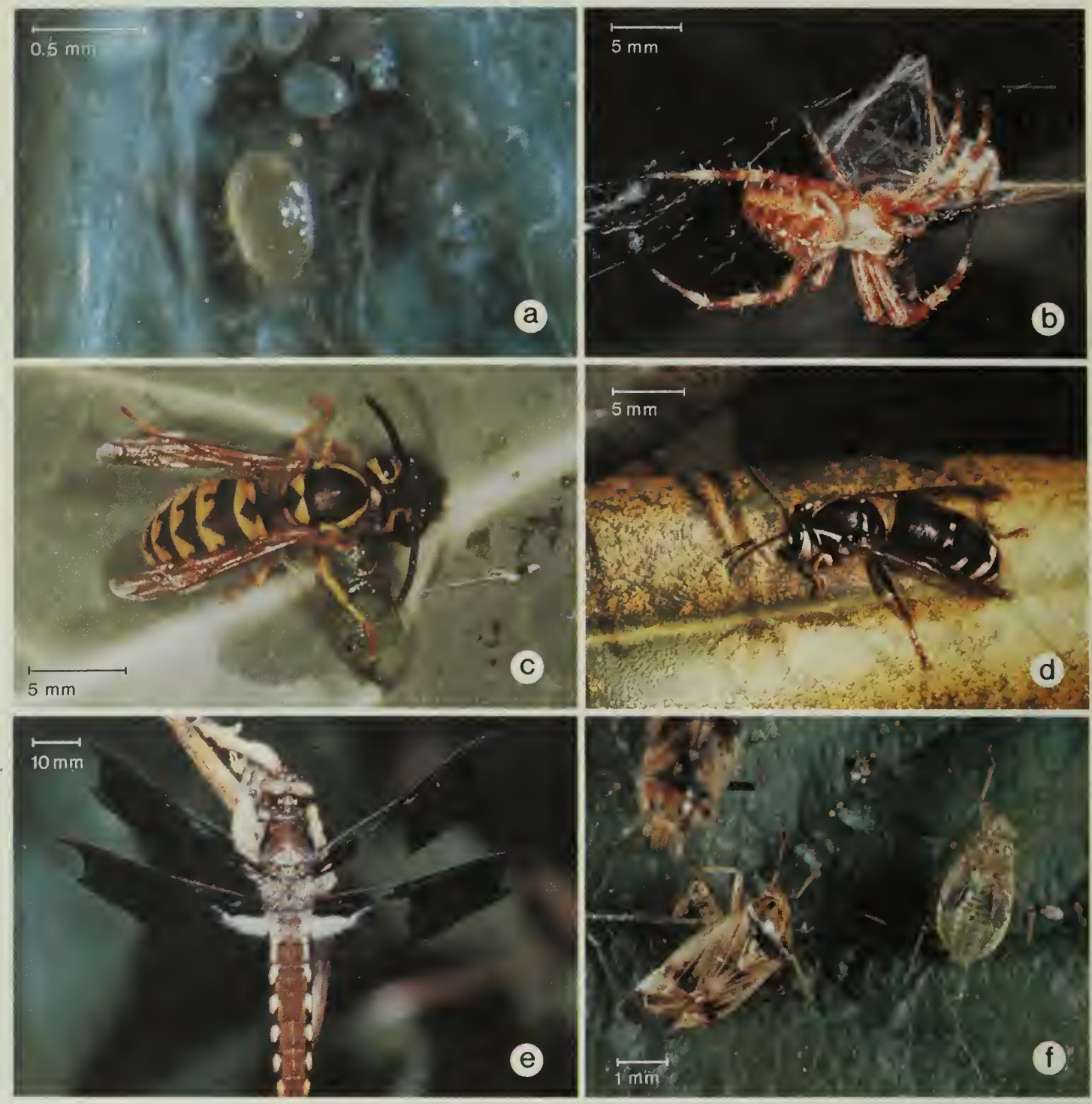

Planche 5 a Phytoséidé et ses œufs (photo D.A. Raworth)

b Araignée et sa proie (photo W.G. Wellington)

c Guêpe jaune et chenille (photo W.G. Wellington)

d Guêpe jaune (photo W.G. Wellington)

e Libellule adulte (photo W.G. Wellington)

$f \quad$ Lygide adulte (avec ailes) et sa nymphe (sans ailes) (photo W. MacDiarmid) 


\section{Névroptères utiles}

\section{Chrysopes (Névroptères : chrysopidés)}

On compte 25 espèces de chrysopes au Canada. Ce prédateur des ravageurs à corps mou est relativement commun et facile à identifier. Long de $14 \mathrm{~mm}$, l'adulte (Chrysopa) a un corps vert doté de grandes ailes membraneuses et d'yeux souvent dorés. Il s'active surtout la nuit (photo de couverture). Attiré par la lumière, il se pose fréquemment sur la vitre des fenêtres éclairées ou se faufile dans les maisons. La femelle pond des œufs caractéristiques blancs et allongés, seuls ou en petits groupes sur les feuilles, chacun à l'extrémité d'un long pédoncule de $10 \mathrm{~mm}$ (planche 4d). La larve brunâtre qui sort de l'œuf puis descend le pédoncule ressemble à un petit alligator poilu aux grosses mâchoires recourbées en forme de pinces. Ces mâchoires servent à attraper les proies et à en extraire tous les liquides organiques (planche 4e). En raison de sa voracité, on l'appelle couramment le lion des pucerons. La larve est un prédateur plus important que l'adulte, car elle peut dévorer d'une vingtaine à quelques centaines de pucerons par jour. Même si elle affectionne les pucerons et les cochenilles, elle ne dédaigne pas les tétranyques, les œufs d'acariens, les cicadelles, les petites chenilles et les thrips. L'adulte de certaines espèces préfère le nectar des plantes et le miellat des pucerons ou des cochenilles, mais beaucoup dévorent également des insectes.

La larve (planche 4e) doit mesurer environ $6 \mathrm{~mm}$ de long avant d'entamer la pupaison. La pupe sphérique ressemble à un cocon blanc au fil très serré, déposé sur le feuillage. Le cycle biologique complet dure de 30 à 40 jours. Durant sa vie de 4 à 6 semaines, la femelle adulte peut pondre de 100 à 200 œufs. La chrysope est le premier prédateur à se manifester au printemps, quand le temps est encore frais. Elle revêt donc une grande importance dans la lutte contre les pucerons au début de la période végétative. Au Canada, on en connaît 25 espèces. On tente actuellement de mettre au point des techniques d'élevage en vue d'utiliser cet insecte dans la lutte biologique contre les pucerons. Il est donc possible qu'on puisse s'en procurer dans le commerce dans un proche avenir.

\section{Hémérobies (Névroptères : hémérobidés)}

L'hémérobie ressemble à la chrysope sauf pour la couleur, qui va du brun au gris brun, et pour la taille, légèrement plus petite (planche $4 f$ ). Ayant des habitudes nocturnes, comme la chrysope, elle pénètre souvent dans les bâtiments éclairés, la nuit, en été. La larve ressemble à celle de la chrysope par la forme et la couleur, mais son appareil buccal est plus court et plus trapu. Les œufs allongés ne sont pas fixés à un pédoncule. Blancs au moment de la ponte, ils virent à l'orange ou au rose puis au brun foncé un peu avant l'éclosion. Au lieu d'un cocon sphérique très dense comme celui de la chrysope, l'hémérobie tisse un cocon lâche, de forme elliptique, dans un endroit abrité plutôt qu'à la vue, sur les feuilles. 
L'hémérobie adulte et sa larve attaquent les petits insectes à corps mou comme les pucerons, de même que tous les stades du tétranyque. La femelle pond plus d'œufs que la chrysope et les larves de l'hémérobie sont encore plus nombreuses que celles de la chrysope dans certains endroits, au début de la saison de croissance. Le cycle biologique dure de 24 à 30 jours. On compte 23 espèces d'hémérobie au Canada.

\section{Acariens utiles}

\section{Acariens prédateurs (Acariens : phytoséidés)}

Les phytoséidés mesurent rarement plus de $0,5 \mathrm{~mm}$ de longueur et sont difficiles à apercevoir à l'œil nu. Avec une loupe cependant, on peut les observer sur les feuilles de fraisier et de framboisier infestées de tétranyques. Souvent globuleux ou piriformes (planche $5 a$ ), ils se déplacent plus rapidement que leurs congénères qui parasitent les végétaux. La différence relative de vitesse est une bonne manière de les reconnaître, mais il en existe de nombreuses espèces et l'identification exige l'œil d'un expert de même qu'un microscope sophistiqué. Une espèce, Amblyseius fallacis (Garman) (planche 5a, p. 24), se retrouve sur tous les fraisiers et framboisiers du Canada, sauf dans les provinces des Prairies.

Les acariens prédateurs se servent de leurs pièces buccales pour trouver, attraper, percer et sucer leurs proies, lesquelles comprennent des acariens, des insectes et des nématodes parasites. Beaucoup d'entre eux se nourrissent également de sève, de miellat (des pucerons), de champignons et de pollen, ce qui permet à leur population de rester stable même quand ils n'ont pas de proies. La femelle de A. fallacis peut pondre de 1 à 3 œufs par jour tandis que d'autres espèces en produisent quotidiennement moins d'un à plus de quatre en moyenne, selon la température et l'abondance de nourriture. Il faut de 5 à 16 jours pour que le cycle de l'œuf à l'adulte s'accomplisse. Aux températures estivales $\left(27^{\circ} \mathrm{C}\right)$, le cycle biologique de nombreuses espèces prédatrices dure environ 6 jours. Les phytoséidés font souvent preuve d'une grande efficacité comme agent de lutte biologique, car ils se reproduisent plus rapidement que les espèces parasites. Il est possible de s'en procurer dans le commerce au Canada où on les utilise principalement pour lutter contre le tétranyque à deux points (Tetranychus urticae Koch).

\section{Autres prédateurs}

Dans des conditions normales, n'importe quelle culture abritera d'autres prédateurs, souvent en grand nombre, mais il ne s'agit pas de prédateurs spécifiques aux insectes qui parasitent la culture. En font partie notamment les araignées tisseuses (planche $5 b$ ) comme l'épeire des jardins et les araignées fouisseuses comme les thomisides. Beaucoup de guêpes entrent également dans ce groupe, comme la guêpe jaune (planche $5 c$ ) et l'espèce Vespula maculata (planche $5 d$ ). Le rôle des asiles, qui attaquent et capturent les insectes en plein vol, n'est pas non plus à négliger. Leurs 
larves, également prédatrices, détruisent les larves des coléoptères (vers blancs) ou leurs pupes sur le sol. Les libellules (planche 5e) et les demoiselles se font parfois nombreuses et chassent activement d'autres insectes.

Enfin, les oiseaux insectivores participent à la lutte contre les ravageurs, non seulement les insectes volants mais les chenilles et les pucerons. Quelques petits mammifères comme la taupe sont des chasseurs opportunistes qui dévorent les gros insectes ou les pupes qu'ils découvrent sur le sol ou sous terre.

\section{Ravageurs courants du framboisier et du fraisier}

\section{Punaises et lygides (Hémiptères : miridés)}

Ce groupe comprend les lygides (punaise terne) (planche $5 f$ ), les pentatomes et d'autres hémiptères. La plupart traversent l'hiver à l'état d'œuf, mais certains adultes hivernent sous les débris végétaux, en plein champ. Au printemps, l'œuf éclôt ou l'adulte se réveille et se met à pondre. La nymphe des lygides ressemble à un gros puceron (planche 5f); elle est délicate, habituellement verte et n'a pas d'ailes bien développées. Elle s'enfuit rapidement lorsqu'on la dérange. La nymphe passera par cinq stades ou mues en l'espace d'environ 5 semaines et se nourrira des sucs de la plante à chaque étape, de même qu'à l'état adulte. Le fait qu'elle se nourrit des inflorescences entraîne une pollinisation inégale à l'origine de la "face de chat» du fraisier et de la "grenaille» du framboisier. L'adulte porte des marques caractéristiques sur le dos et ses longues pièces buccales, suceuses, pointues, sont repliées sous l'abdomen. Quand on le dérange, l'insecte s'envole ou s'enfuit pour se mettre à l'abri.

Les insectes de ce groupe ne sont pas tous nuisibles, certains sont des prédateurs. Les cinq espèces qui vivent sur le fraisier s'attaquent toutes à la plante, mais l'une d'entre elles se nourrit également d'autres insectes. Parmi les 22 espèces qu'on retrouve sur le framboisier au Canada, 6 se nourrissent de la plante, 10 sont des prédateurs et 6 alternent les deux régimes.

Les lygides sont parasités par plusieurs guêpes de la famille des braconidés, au moins un ichneumon et plusieurs tachinaires. On a recensé beaucoup de prédateurs des lygides, notamment des punaises demoiselles, des punaises tête-de-clou, des miridés et des chrysopes, mais on ignore l'importance de leur rôle. Ces prédateurs préfèrent habituellement les insectes à corps mou. Toutefois, ils s'attaqueront également aux lygides, et plus particulièrement à leurs jeunes nymphes.

\section{Pucerons (Homoptères : aphididés)}

Les pucerons sont de petits insectes à corps mou qui sucent la sève des plantes. Ils sont habituellement de couleur verte, noire, jaunâtre ou rose. Durant la majeure partie de la période végétative, ils déposent leurs jeunes vivants sur la plante dont ils se nourrissent. Ce comportement crée des amas ou colonies de pucerons de tout âge (planche $6 a$, p. 28). Quand il se 

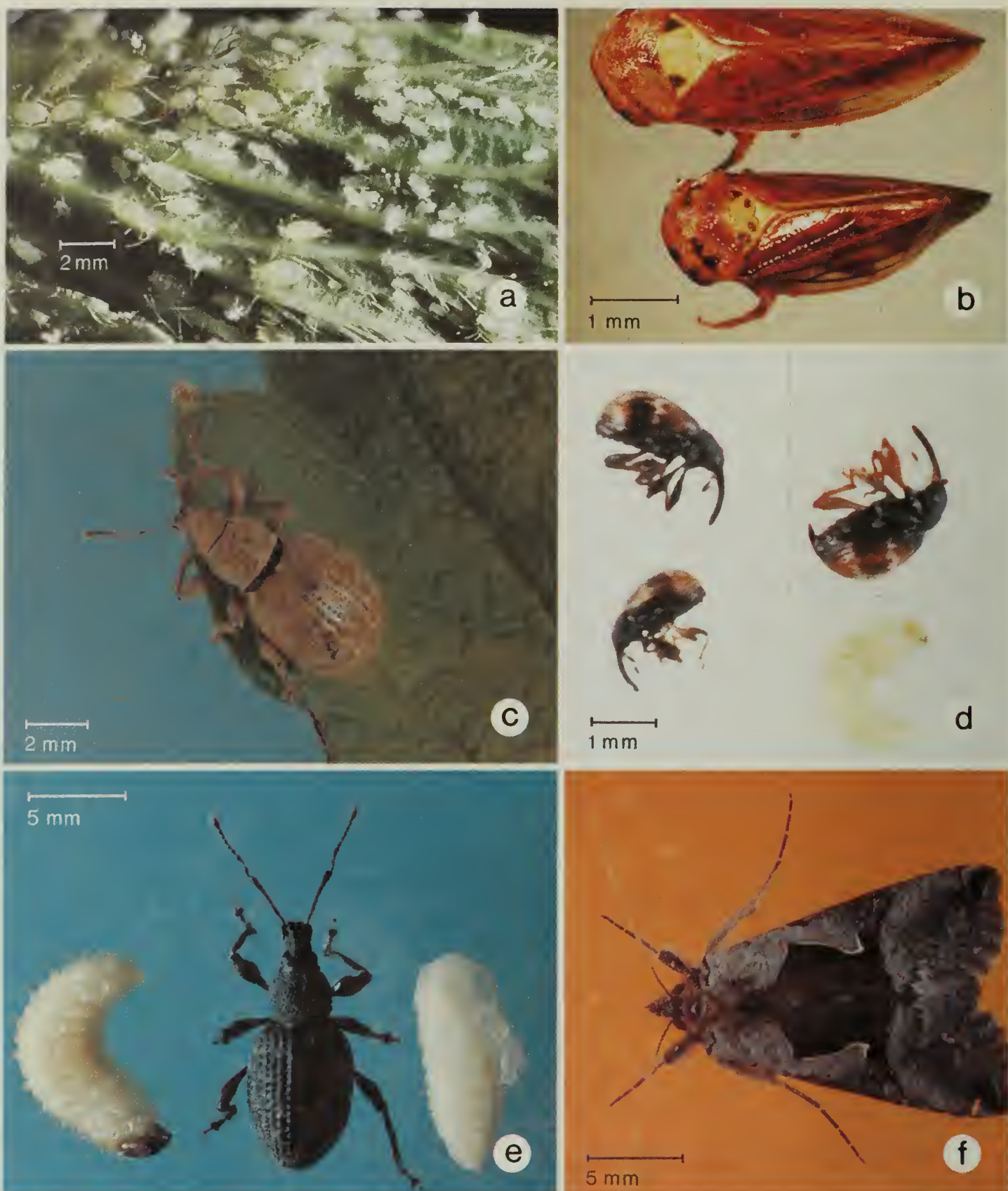

d

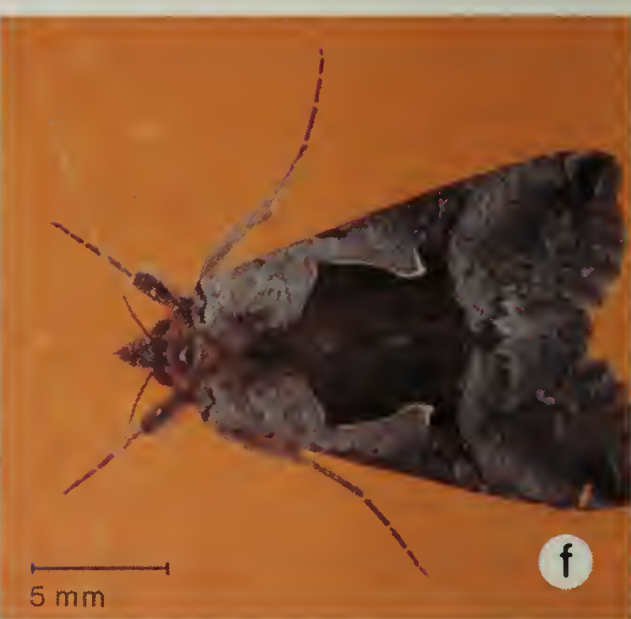

Planche 6 a Pucerons sur une feuille de fraisier (photo W. MacDiarmid)

b Cicadelle du genre Macropsis (sur un framboisier) (photo H. Severson)

c Charançon sur une feuille de fraisier (photo W. MacDiarmid)

d Anthonome de la fleur du fraisier adulte et sa larve (photo N. Bostanian)

e Charançon noir de la vigne adulte, sa larve et sa pupe (photo W.T. Cram)

$f$ Arpenteuse du framboisier (Autograpita ampla) (Walker)) (photo W. MacDiarmid) 

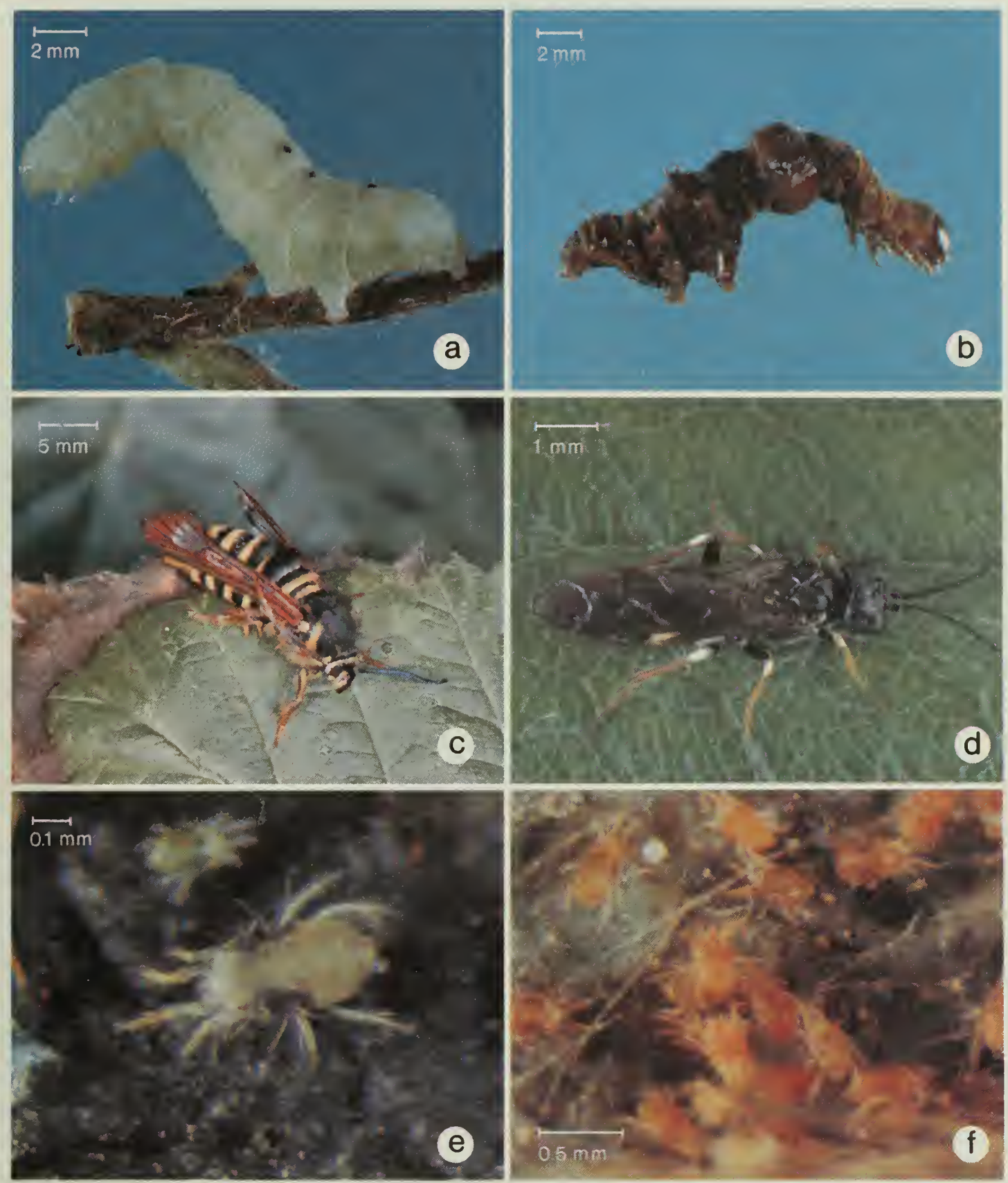

Planche 7 a Arpenteuse du framboisier parasitée (remarquer les taches noires) (photo W. MacDiarmid)

b Dépouille d'arpenteuse parasitée (photo W. MacDiarmid)

c Rhizophage du framboisier adulte (photo W.G. Wellington)

d Tenthrède adulte (photo W.T. Cram)

$e$ Tétranyque à deux points adulte (photo C. Chan)

$f$ Femelle rouge du tétranyque à deux points, hivernant (photo C. Chan) 
nourrit, le puceron aspire tant de sève qu'il doit l'excréter rapidement, sinon il risquerait d'éclater. On appelle miellat le liquide ainsi excrété, car il renferme une grande quantité des sucs de la plante. Cette substance luisante et collante tache les végétaux près des colonies. En trop grande quantité, le miellat favorisera l'apparition d'une moisissure fuligineuse qui contaminera encore plus la plante. Le miellat attire aussi les prédateurs et les parasites des pucerons pour deux raisons : en premier lieu, beaucoup de prédateurs découvrent les pucerons en cherchant le miellat et deuxièmement, les sucres et les acides aminés que contient celui-ci constituent une bonne source d'éléments nutritifs (p. ex. pour les chrysopes adultes, les guêpes parasites, les syrphes adultes, les cécidomyies et les coccinelles).

Le puceron du framboisier, avec ses $3 \mathrm{~mm}$ de longueur, est passablement gros. Son corps va du vert foncé au jaune et il affectionne les jeunes pousses terminales. Le puceron du fraisier (planche $6 a$ ) est plus petit et vert pâle, et porte de nombreux poils. Il hiverne à l'état adulte ou sous forme de petits œufs noirs, sur les vieilles feuilles du plant. Ces deux espèces revêtent une grande importance, car elles transmettent des viroses à la plante.

Le puceron est la nourriture préférée de plusieurs insectes prédateurs comme les syrphes, les cécidomyies, les chrysopes, les coccinelles et quelques staphylins. Beaucoup d'autres insectes prédateurs, les punaises demoiselles, les réduves et les carabes par exemple, les détruisent également. Enfin, ils sont attaqués par maintes espèces de guêpes parasites, surtout celles de la famille des braconidés.

\section{Cicadelles et cercopes (Homoptères)}

Les cicadelles, qui s'attaquent au framboisier, et les cercopes, qui peuvent causer des ravages au fraisier, sont apparentés aux pucerons et détériorent les plantes un peu de la même manière, en aspirant la sève. La cicadelle est un insecte de $3 \mathrm{~mm}$ de longueur, très actif, au corps élancé qui fait un saut lorsqu'on le dérange. La couleur du corps varie du blanc pâle au vert brunâtre (planche 6b) et l'insecte se nourrit sur la face inférieure des feuilles de framboisier. Leur activité fait apparaitre des taches jaunes le long des nervures et à l'extrémité des feuilles, ce qui leur donne un aspect marbré. Certaines espèces se nourrissent également des baies. La cicadelle apparaît vers la fin mai dans certaines régions, mais ailleurs on ne la voit qu'en juin et en juillet. Comme le puceron, la cicadelle excrète du miellat qui macule la plante ou le fruit d'une substance luisante et collante sur laquelle peut apparaître une moisissure fuligineuse. À l'instar des pucerons, la cicadelle est la cible de divers prédateurs comme les chrysopes, les coccinelles, certains staphylins, les punaises demoiselles, les réduves et les carabes.

On trouve des cercopes un peu partout au Canada. Quand ils se nourrissent de la sève des fraisiers, ils entraînent le nanisme de la plante et en réduisent le rendement. Cet insecte est comme recouvert de crachat, mélange d'air et d'excréments essentiel à la survie de la nymphe. L'espèce 
en forme de coin qui parasite le fraisier (Philaenus leucophthalmus Linnaeus) a une nymphe de couleur pâle, blanchâtre à jaune, mais devient brun grisâtre à l'état adulte. L'adulte mesure $6 \mathrm{~mm}$ de longueur et vagabonde d'une plante à l'autre en parcourant de brèves distances par vol ou par saut. La femelle pond ses œufs orange sur la plante où la nymphe éclôt au printemps. Il n'existe pas de bon agent de lutte biologique contre les cercopes.

\section{Charançons et autres coléoptères (Coléoptères)}

Plusieurs charançons s'attaquent aux racines, à la couronne, aux bourgeons et aux feuilles du framboisier et du fraisier (planche 6c). Les dommages les plus importants surviennent quand la larve détruit les racines (habituellement de 5 à $15 \mathrm{~cm}$ sous la surface) ou la couronne. La majorité des charançons qui parasitent le fraisier et le framboisier se reproduisent de façon asexuée (sans mâle). Aucune espèce ne vole. L'adulte s'active la nuit, si bien qu'on l'aperçoit rarement durant le jour. On peut néanmoins déceler sa présence par les coches caractéristiques qui découpent la bordure des feuilles ou, dans le cas de l'anthonome de la fleur du fraisier (planche $6 d$ ), par les bourgeons floraux sectionnés. La larve (planches $6 d$ et $e$ ) vit dans le sol et se nourrit des racines durant toute la période végétative. Les dommages que la larve cause à la couronne entraînent le flétrissement de la plante à la nouaison. Le plus gros charançon est le charançon noir de la vigne (Othiorhynchus sulcatus (Eichoff)) qui mesure $13 \mathrm{~mm}$ (planche 6e). Le plus petit, le charançon des bois (Nemocestes incomptus (Horn)), n'a que la moitié de cette taille.

Il est capital de différencier les charançons adultes des carabes utiles, car certains se ressemblent par la taille et la couleur. Le charançon se distingue par son «nez». En effet, la tête de l'adulte, plus étroite, termine un thorax allongé qui imite un cou; ses antennes sont couramment pliées au centre. Le corps est dur et de couleur terne et l'insecte se déplace assez lentement. $\mathrm{Au}$ contraire, les carabes prédateurs fuient à toute vitesse lorsqu'on les dérange. Ils ont plutôt une forme arrondie, de même qu'une carapace luisante. Ces deux types d'insectes sont nocturnes et passent la journée sous des débris ou dans des galeries.

Les charançons ne sont pas la proie d'autant d'insectes que les pucerons, mais divers vertébrés (p. ex. les taupes) de même que de plus gros carabes et des staphylins en font leurs proies. Plusieurs petits carabes mangent également les œufs des charançons. D'autres prédateurs comme les perce-oreilles s'attaquent à divers stades du charançon en laboratoire, mais on ignore leur rôle véritable dans la nature.

Divers coléoptères parasitent le framboisier et le fraisier. Le byture de la framboise existe un peu partout au Canada. L'adulte mange les jeunes feuilles et les inflorescences, ce qui réduit la nouaison. De son côté, la larve pénètre dans le fruit et provoque sa chute ou le rend impropre à la commercialisation. Ce ravageur subit les attaques des mêmes prédateurs que le charançon. La chrysomèle du fraisier est un autre coléoptère parasite qu'on trouve aussi fréquemment sur le fraisier et le framboisier. 
L'adulte se nourrit des feuilles de la plante la nuit tandis que la larve en dévore les racines et la couronne. Les deux sont la cible des prédateurs qui vivent dans le sol comme les staphylins et les carabes.

\section{Enrouleuses, chenilles, noctuelles et perceurs (Lépidoptères)}

Ce groupe se compose de papillons nocturnes (planche $6 f$ ) dont la forme immature, une chenille, dévore différents organes de la plante : feuilles, couronne, racines et tige. Plusieurs espèces parasitent le fraisier et le framboisier à divers degrés, dans toutes les régions du Canada. Il s'agit d'un groupe diversifié de ravageurs auxquels s'attaquent des prédateurs et des parasites très variés également (tachinaires, ichneumons, braconides, chalcidies, carabes, réduves, guêpes comme la guêpe jaune et, dans une moindre mesure, les staphylins, les coccinelles, les punaises demoiselles, les anthocoris, les chrysopes et d'autres prédateurs non spécifiques comme les oiseaux et les rongeurs). En règle générale, une chenille parasitée par une guêpe ou un tachinaire porte les stigmates causés par l'inoculation des œufs (planche 7a, p. 29). À sa mort, elle laisse souvent une dépouille typique qu'illustrent les séquelles du parasitisme (planche $7 b$ ).

Quoique le rhizophage du framboisier (Bembicia marginata (Harris)) soit un papillon nocturne, il ressemble et se comporte comme une guêpe jaune (planche 7c). Ce mimétisme le met à l'abri des prédateurs comme les oiseaux qui le prennent pour une guêpe et ne s'y frôlent pas de peur de se faire piquer. Le papillon nocturne adulte volette dans les framboisiers et dépose des œufs sur la face inférieure des feuilles durant la journée, de la fin juillet à la mi-septembre. De chaque œuf émerge une petite larve jaune qui longe la ronce jusqu'au sol. Elle creuse ensuite une petite ouverture dans l'écorce et y passe l'hiver. Le printemps suivant, la larve s'enroule autour d'une nouvelle tige avant de percer une galerie jusqu'au cœur tendre et creux de la ronce qui lui servira de grenier et d'abri pour le deuxième hiver. Le printemps venu, la larve, qui a grossi, se creuse un chemin à travers la tige jusqu'à quelques centimètres au-dessus du sol. La pupaison commence immédiatement sous l'écorce. Le papillon adulte fait son apparition à la fin de juillet.

En raison de sa ressemblance avec les syrphes adultes et la guêpe jaune, le rhizophage du framboisier est rarement considéré comme un ravageur du framboisier. De même, on peut confondre les guêpes et les syrphes avec les rhizophages. Le corps du papillon est cependant recouvert d'écailles plumeuses qui lui donne une apparence veloutée et mate tandis que le corps des syrphes et de la guêpe jaune a une texture lisse et luisante.

\section{Tenthrèdes (Hyménoptères : tenthrèdinidés)}

Le tenthrède du framboisier (Monophadnoides geniculatus (Hartig)) adulte est une guêpe noire, aux ailes translucides, marquée de rouge et de jaune (planche $7 d$ ). Son corps trapu d'environ $6 \mathrm{~mm}$ de longueur n'a pas la taille étroite qui est typique des guêpes. Elle se manifeste en mai ou en juin et dépose ses œufs sur la feuille terminale des ronces de l'année. De l'œuf 
sort une larve verte et épineuse, difficile à percevoir, car elle se confond avec le feuillage. Dans les infestations graves, la larve dévore le tissu foliaire pour ne laisser que les plus grosses nervures. À maturité, elle mesure de 12 à $17 \mathrm{~mm}$ de longueur et se laisse choir sur le sol où elle tisse un cocon dans lequel elle passera l'hiver. Les braconides attaquent les œufs du tenthrède, tandis que le cocon est une proie facile pour les carabes et les staphylins. Enfin la larve constitue une cible idéale pour les ichneumons et les tachinaires, de même que la guêpe jaune et les oiseaux.

\section{Acariens}

Le tétranyque à deux points (Tetranychus urticae Koch) adulte est difficile à voir à l'œil nu. Il s'agit d'un insecte jaune verdâtre portant deux taches foncées sur le dos (planche 7e). Il se nourrit en suçant la sève des plantes sur la face inférieure des feuilles. Les feuilles parasitées sont mouchetées de blanc ce qui leur donne un aspect terne et pâle. Lorsque l'infestation est grave, la feuille brunit et finit par tomber. Le tétranyque tisse une toile soyeuse sur la face inférieure des feuilles dont il se nourrit. La femelle rouge orange passe l'hiver à l'état adulte (planche $7 f$ ) sur les feuilles ou sous les détritus, les feuilles mortes ou le sol au pied du plant. Au printemps, elle trouve une jeune feuille dont elle se nourrira et sur laquelle elle déposera ses œufs. La population de tétranyques à deux points augmente par temps chaud et sec. Il est possible aussi que la poussière qui macule les framboisiers et les fraisiers en bordure des chemins fasse augmenter la population des tétranyques à deux points.

L'acarien du cyclamen ou du framboisier (Phytonemus pallidus (Banks)) est un acarien minuscule qui se nourrit dans la couronne et les feuilles du fraisier avant qu'elles se déroulent. S'il est difficile à distinguer même avec une loupe, il n'en va pas de même pour les dommages qu'il cause. En effet, les feuilles jaunissent et ne se déroulent pas; celles qui parviennent à s'ouvrir ont habituellement l'aspect du cuir et sont difformes. Les plants fortement infestés sont souvent rabougris et lorsque le ravageur s'attaque à l'inflorescence, les fruits deviennent difformes. Les acariens prédateurs comme les espèces du genre Amblyseius jouent un grand rôle dans la lutte contre ce parasite.

Sur la côte ouest, un ériophyide appelé phytopte du toyon (Acalitus essigi (Hassan)) cause d'importants ravages aux framboisiers. Cet acarien est microscopique; il passe l'hiver dans les bourgeons et dévore le fruit durant son développement au printemps et en été. Les baies touchées restent dures et sûres, même après maturité. Les acariens prédateurs permettent de combattre ce ravageur dans une certaine mesure.

Les acariens comptent beaucoup de prédateurs : chironomes, petites coccinelles noires du genre Stethorus, quelques petits staphylins, punaises tête-de-clou, anthocoris, chrysopes et hémérobies ainsi que d'autres acariens. Dans le sud de l'Ontario, le thrips à six taches, Scolothrips sexmaculatus (Pergande), s'attaque aux tétranyques. On le trouve couramment aux États-Unis, mais il n'a pas souvent été signalé au Canada. 


\section{Bibliographie}

Borrer, D.J.; DeLon, D.M.; Triplehorn, C.A. 1976. An introduction to the study of insects. $4^{\circ}$ édition. Holt Reinhart \& Winston.

Carr, A. 1979. Rodale's color handbook of garden insects. Rodale Press, Erasmus, $\mathrm{Pa}$.

Chant, D.A. 1985. External anatomy. Page 5-9 in Helle, W.; Sabelis, M.W., eds. Spider mites and their biology, natural enemies and control. Vol. 1B. Elsevier, Amsterdam.

Chant, D.A.; Hansell, R.I.C. 1971. The genus Amblyseius (Acari: Phytoseiidae) in Canada and Alaska. Can. J. Zool. 49:703-758.

Clausen, C.P. 1940. Entomophagous insects. McGraw Hill Biik Co., N.Y.

Clancy, D.W.; Pierce, H.D. 1966. Natural enemies of some Lygus bugs. J. Econ. Entomol. 59:853-858.

Danks, H.V. 1979. Canada and its insect fauna. Entomol. Soc. Can. Memoir 108.

Davidson, R.H.; Lyon, W.F. 1979. Insect pests of farm, garden, and orchard. $7^{\circ}$ édition. John Wiley and Sons, N.Y.

Garth, G.S.; Shanks, C.H., Jr. 1978. Some factors affecting infestation of strawberry fields by the black vine weevil in western Washington. J. Econ. Entomol. 71:443-448.

Gordon, R.D. 1985. The Coccinellidae (Coleoptera) of America north of Mexico. J. N.Y. Entomol. Soc. 93:1-912.

Kelton, L.A. 1982. Plant bugs on fruit crops in Canada. Research Branch, Agric. Can. Monograph No. 24.

Lattin, J.D. 1989. Bionomics of the Nabidae. Ann. Rev. Entomol. 34:383-400.

Lim, K.P.; Stewart, R.K. 1976. Parasitism of the tarnished plant bug, Lygus lineolaris (Hempitera: Miridae) by Peristenus pallipes and $P$. pseudopallipes (Hymenoptera: Braconidae). Can. Entomol. 108:601-608.

McMurtry, J.A.; Hummaker, C.B.; van de Vrie, M. 1970. Ecology of tetranychid mites and their natural enemies: A review. I. Tetranychid enemies: Their biological characters and the impact of spray practices. Higardia 40:331-390.

Oatman, E.R.; Badgley, M.E.; Platner, G.R. 1985. Predators of the two-spotted spider mite on strawberry. Calif. Agric. janvier/février:9-12.

Putman, W.L. 1955. The bionomics of Stethorus punctillum Weise (Coleoptera: Coccinellidae) in Ontario. Can. Entomol. 87:9-33.

Schneider, F. 1969. Bionomics and physiology of aphidophagous syrphidae. Ann. Rev. Entomol. 14:103-124. 
Shanks, C.H., Jr. 1981. Insects and mites. Pages 293-308 in Childers, N.F., ed. The strawberry. Horticultural Publications, Gainsville, Fla.

Tanigoshi, L.K. 1982. Advances in knowledge of the biology of the Phytoseiidae. Pages 1-22 in Hoy, M.A., ed. Recent advances in knowledge of the Phytoseiidae. Univ. Calif. Press Publ. 3284.

Ware, G.W. 1980. Complete guide to pest control-with and without chemicals. Thomson Publications, Fresno, Calif.

\section{Publications des provinces et des États}

Colombie-Britannique. 1988. Berry production guide for commercial growers. Ministère de l'Agriculture et des Pêcheries de la Colombie-Britannique, British Columbia Blueberry Co-op Association, British Columbia Cranberry Marketing Board, British Columbia Raspberry Growers Association et Fraser Valley Strawberry Growers Association.

Hunter, C.L. 1981. Insects and diseases of cane fruits. Ministère de l'Agriculture et de l'Alimentation de l'Ontario. Feuillet No. 81-001, Agdex 230.605.

Johansen, C.; Eves, J.; Retan, A. 1972. Beneficial predators and parasites found on Washington crops. Washington State University Extension, Extension Bulletin 640.

Raspberry pest identification. 1988. Plant Industry Branch, Ministère de l'Agriculture du Nouveau-Brunswick. Publ. No. 020-88, Agdex No. 232.600 .

Retan, A.H.; Curtis, J. 1972a. Insect answers - minute pirate bug. Co-operative Extension Service, College of Agriculture, Washington State University, Pullman, E.M. 3703.

Retan, A.H.; Curtis, J. 1972c. Insect answers - hover or syrphid flies. Co-operative Extension Service, College of Agriculture, Washington State University, Pullman. E.M. 3704.

Strawberry pest identification. 1988. Plant Industry Branch, Ministère de l'Agriculture du Nouveau-Brunswick. Publ. No. 010-88, Agdex No. 232.600 .

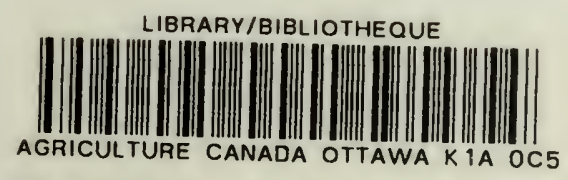

3907300080589 ? 


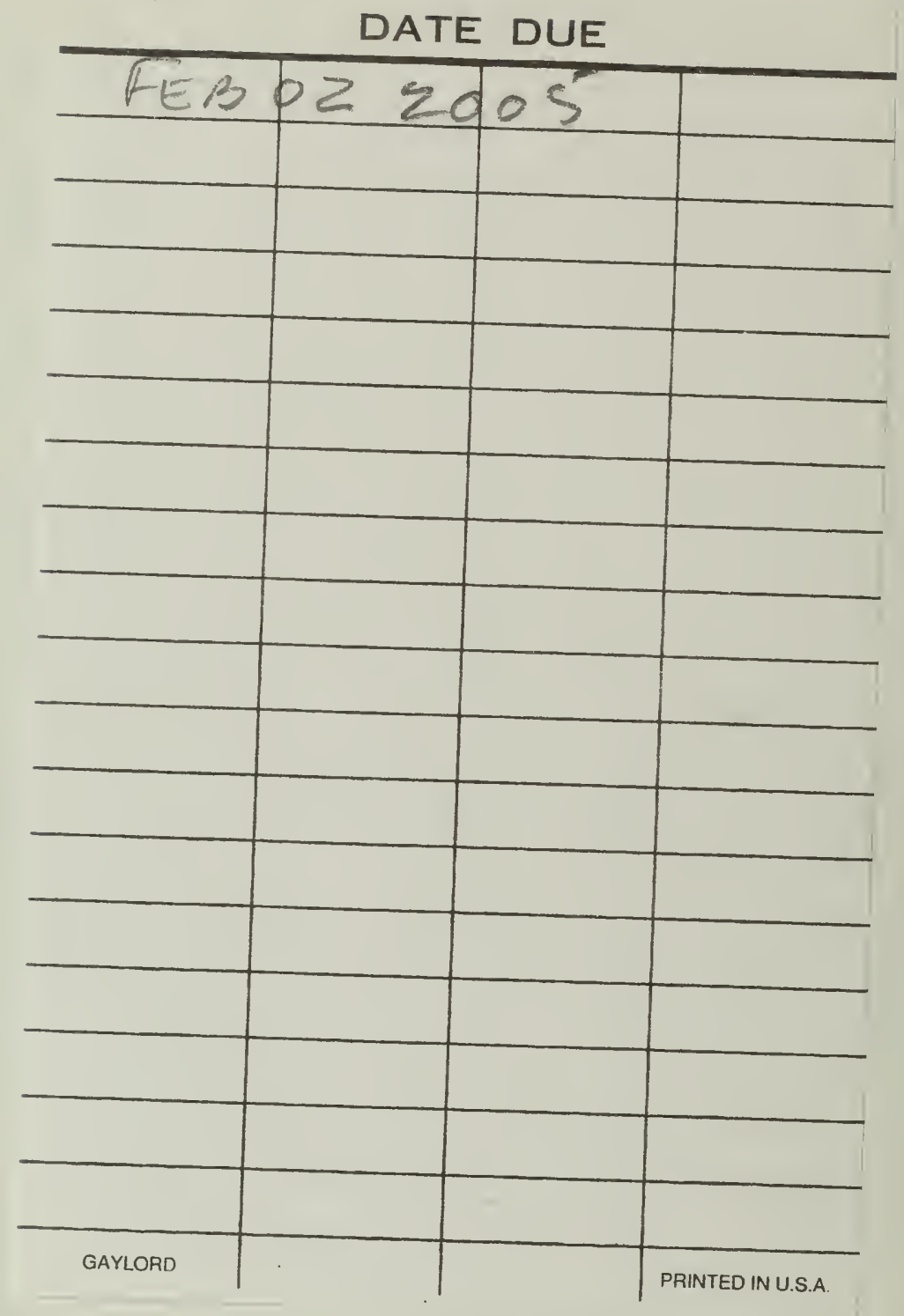

Papier
recyclé 